\title{
Flow structure in the wake of a space-launcher model with propulsive-jet simulation
}

\author{
Anne-Marie Schreyer ${ }^{1,2}$
}

Received: 13 November 2019 / Revised: 12 February 2020 / Accepted: 24 February 2020 / Published online: 17 March 2020

(c) The Author(s) 2020

\begin{abstract}
The flow around a space launcher is dominated by flow separation at the junction between the main body and engine and strongly influenced by the propulsive jet. The flow field is highly unsteady with strong local pressure and temperature loads. To conceive a means of mitigating these effects and allow for smarter launcher designs, a better understanding is necessary. The wake of a generic axisymmetric launcher model with and without afterexpanding propulsive jet was investigated at Mach 2.9, a Reynolds number $\operatorname{Re}_{D}=1.3 \cdot 10^{6}$ (model diameter $\mathrm{D}$ ), and a nozzle exit velocity of the jet simulation of Mach 2.5. I observed the evolution of the flow and its spectral content with velocity measurements with particle image velocimetry and wall-pressure recordings. Coherent structures were analyzed based on a proper orthogonal decomposition. The propulsive jet has two major influences. First, the turbulent intensities in the shear layer are damped, and the larger structures in the wake contain less turbulent kinetic energy than in the baseline case without propulsive jet. Second, the reattachment process is modified and the wake instability phenomena change accordingly. Without propulsive jet, large-scale separation with reattachment on the nozzle fairing occurs and the low-frequency unsteadiness of the closed separation bubble/recompressionshock system is relevant. This phenomenon only has a minor influence under the influence of the jet plume, where the flow is displaced away from the surface and the reattachment process is incomplete. Here, shear-layer instabilities become more prominent.
\end{abstract}

Keywords Unsteadiness · Supersonic wake flow · Generic space-launcher model · Propulsive-jet simulation

\begin{tabular}{|c|c|c|c|}
\hline & \multirow[t]{2}{*}{$V$} & \multirow{2}{*}{$\begin{array}{l}\text { Mean velocity component in the radial direction (y } \\
\text { direction) }[\mathrm{m} / \mathrm{s}]\end{array}$} \\
\hline$D$ & Diameter of space-launcher model main body & & \\
\hline$d$ & Diameter & \multirow[t]{2}{*}{$u$} & \multirow{2}{*}{$\begin{array}{l}\text { Turbulence intensity of the velocity component in } \\
\text { the axial direction ( } x \text { direction) }[\mathrm{m} / \mathrm{s}]\end{array}$} \\
\hline & Frequency $[\mathrm{Hz}]$ & & \\
\hline$M$ & Mach number & $u_{d}$ & Discretization velocity $[\mathrm{m} / \mathrm{s}]$ \\
\hline$M_{\mathrm{Mol}}$ & Molar mass & \multirow[t]{2}{*}{$v$} & \multirow{2}{*}{$\begin{array}{l}\text { Turbulence intensity of the velocity component in } \\
\text { the radial direction ( } y \text { direction) }[\mathrm{m} / \mathrm{s}]\end{array}$} \\
\hline$n$ & Number of POD mode & & \\
\hline$p$ & Pressure & $x$ & Direction along model length axis (axial direction) \\
\hline $\begin{array}{l}\operatorname{Re}_{D} \\
r\end{array}$ & $\begin{array}{l}\text { Reynolds number (based on D) } \\
\text { Radius of the launcher-model main body }\end{array}$ & $y$ & $\begin{array}{l}\text { Radial direction on opposite site of model strut } \\
\text { support }\end{array}$ \\
\hline $\begin{array}{l}S t_{D} \\
T\end{array}$ & $\begin{array}{l}\text { Strouhal number (based on D) } \\
\text { Temperature }\end{array}$ & $\Delta x_{\max }$ & $\begin{array}{l}\text { Maximum streamwise particle displacement } \\
\text { [pixels] }\end{array}$ \\
\hline$U$ & $\begin{array}{l}\text { Mean velocity component in the axial direction ( } x \\
\text { direction) }[\mathrm{m} / \mathrm{s}]\end{array}$ & $\begin{array}{l}\Delta \tau \\
\delta\end{array}$ & $\begin{array}{l}\text { Temporal delay between two images }[\mu \mathrm{s}] \\
\text { Boundary-layer thickness }[\mathrm{mm}] \\
\text { PIV calibration [pixels } / \mathrm{mm}]\end{array}$ \\
\hline \multirow{2}{*}{\multicolumn{2}{|c|}{$\begin{array}{l}\triangle \text { Anne-Marie Schreyer } \\
\text { a.schreyer@aia.rwth-aachen.de }\end{array}$}} & $\phi_{n, i}$ & $\begin{array}{l}\text { Spatial POD mode number } n \text { for axial }(i=x) \text { or } \\
\text { radial }(i=y) \text { velocity fluctuation component }\end{array}$ \\
\hline & & $\kappa$ & Heat capacity ratio \\
\hline & nschweig, 38108 Braunschweig, Germany & \multirow{2}{*}{$\begin{array}{l}\lambda_{n} \\
\theta\end{array}$} & \multirow{2}{*}{$\begin{array}{l}\text { Eigenspectrum of mode } n \\
\text { Angle specifying the location over the circumfer- } \\
\text { ence of the model }\end{array}$} \\
\hline & $\begin{array}{l}\text { Address: Chair of Fluid Me } \\
\text { ynamics, RWTH Aachen L }\end{array}$ & & \\
\hline
\end{tabular}




$\begin{array}{ll}\mathfrak{R} & \text { Gas constant } \\ \sum_{n} & \begin{array}{l}\text { Cummulative relative energy of the first } n \text { POD } \\ \text { modes }\end{array}\end{array}$

$\begin{array}{ll}\text { Subscript } \\ e & \text { Conditions at nozzle exit (of the TSA) } \\ \text { max } & \text { Maximum value } \\ \text { RK } & \text { Conditions in the settling chamber of the HLB } \\ \mathrm{rms} & \text { Root-mean-square value } \\ \mathrm{SC} & \text { Conditions in the settling chamber of the TSA } \\ \mathrm{SP} & \text { Conditions in the storage tube } \\ t & \text { Total (temperature or pressure) } \\ \infty & \text { Quantity at freestream conditions }\end{array}$

\section{Introduction}

The afterbody geometry of a classical space launcher is characterized by an abrupt step decrease in diameter at the junction between the main body and rocket engine, which causes the separation of the turbulent boundary layer on the main body at the shoulder of the launcher base. A large recirculation region forms downstream of the step and a turbulent shear layer develops. This separation-dominated flow field is highly unstable and induces strong wall-pressure oscillations, which can excite structural vibrations detrimental to the launcher [1]. A better understanding of the flow field is thus crucial to lay the groundwork for minimizations of such detrimental effects and to bring us one step closer toward an efficient launcher design.

The conditions and topology of the wake flow vary enormously along the flight trajectory of the launcher. In part, this is due to the influence of the propulsive jet, which becomes more and more underexpanded with altitude. The subsequent afterexpansion strongly influences the wake flow of the launcher. Depending on the stage in the flight trajectory, the dimensions of the afterbody, and the interaction with the jet plume, the flow may or may not reattach onto the outer wall of the engine nozzle, causing different mean and turbulent flow topologies. For this reason, experimental and numerical studies covering a wide range of flow Mach numbers have been performed. In the transonic range, Deprés et al. [1], Deck and Thorigny [2], and Weiss et al. [3] have investigated cases both with and without a propulsive jet. In supersonic flow, such cases have been studied by Bannink et al. [4], Bourdon and Dutton [5], Janssen and Dutton [6], and Stephan et al. [7, 8], for example. Saile et al. [9, 10] and Statnikov et al. [11] have studied hypersonic cases. These studies describe the mean flow topology and survey base-pressure fluctuations including dominant frequencies or observe the formation of turbulent structures in the wake. van Gent et al. [12] compare the influence of a jet plume and of nozzle length on the wake-flow topology in transonic and supersonic cases.

The large recirculation zone forming around the nozzle causes an increase in pressure at the main body base [11] This coincides with an increased base-pressure fluctuation level, so that structural vibrations are excited that may be critical for the launcher. Simulating the flight conditions of space launchers at higher altitudes, the propulsive jet in supersonic flow is usually underexpanded at the nozzle exit, thereby leading to an afterexpanding jet plume that has a strong displacement effect on the outer flow. If, as a consequence, the flow does not reattach to the nozzle fairing, also thermal loads can become a problem: hot gases from the propulsive jet may be convected upstream in the separation zone, potentially harming the structure. The chosen test cases, namely a supersonic wake flow of an axisymmetric model with and without propulsive jet, are thus critical with respect to structural loads and therefore of special interest.

It is crucial to identify the sources of such potentially detrimental base-pressure fluctuations and eventually understand the governing mechanisms in the turbulent wake-flow field.

Recently, progress has been made in the source identification of pressure fluctuations at specific characteristic frequencies. Janssen and Dutton [6] have experimentally investigated a supersonic base flow at Mach 2.46 and described wall-pressure fluctuations at a dominant frequency of a Strouhal number of $\mathrm{St}_{D}=0.1$ (based on main body diameter D), which they attributed to large turbulent structures in the separated region. These structures in the developing shear layer are altered under the influence of an afterexpanding jet plume [6,11].

Deprés et al. [1] investigated several generic launcher configurations in transonic flow experimentally and found that the shedding of large-scale structures from the separation bubble affects the wall-pressure fluctuations. The shedding is governed by interactions between the structures formed in the shear layer and the wall in the reattachment region. The characteristic frequency for this phenomenon is around $\mathrm{St}_{D}=0.2$ and has been observed in the signal for both reattaching and non-reattaching flow, as well as for cases with and without supersonic propulsive jet. These findings were later confirmed by Deck and Thorigny [2] with their numerical approach.

Statnikov et al. [11] performed a sparsity-promoting dynamic mode decomposition combined with statistical analysis on a zonal RANS/LES simulation of a generic launcher model at Mach 6 and identified the radial flapping motion of the shear layer as the source of a dominant peak at $\mathrm{St}_{D}=0.85$. The swinging motion of the shear layer was found to contribute to the signal with a dominant peak at $\mathrm{St}_{D}=0.6$. 
With this study, I intend to contribute to the understanding of the flow structure and governing mechanisms in the supersonic stage of the flight trajectory by providing detailed surveys of the mean and turbulent flow topologies in the wake of a generic space-launcher model. Furthermore, I investigate the influence of a moderately underexpanded propulsive jet on the wake flow in question. The chosen approach is to survey and discuss the turbulent velocity components and the turbulence structure in the developing shear layer together with the "footprint" of the turbulent structures on the pressure signal on the afterbody. To achieve this, I measured the mean and turbulent velocity fields in the wake flow together with the time-resolved pressure fluctuations at the main body base and on the nozzle fairing. Coherent vortical structures throughout the flow field were identified with proper orthogonal decomposition (POD). By directly comparing these quantities for cases with and without propulsive jet, conclusions regarding the modification of the mechanisms governing the flow behavior under the influence of the jet plume can be drawn. This completes the knowledge obtained from previous studies, where such quantities and effects were investigated separately or for different flow regimes.

I studied the wake of an axisymmetric generic spacelauncher model for two flow cases: a baseline case without propulsive jet and a jet-simulation case with a cold air jet. Experimental investigations were performed in the hypersonic ludwieg tube Braunschweig (HLB) in supersonic configuration [13] at a flow Mach number of 2.9 and a Reynolds number $\operatorname{Re}_{D}=1.3 \cdot 10^{6}$ based on model diameter $\mathrm{D}$. This corresponds to a simulated trajectory point at $25 \mathrm{~km}$ altitude. Pressure fluctuations at the main body base were measured with Kulite pressure transducers, while the velocity field in a plane covering the development region of the shear layer in the wake was measured with particle image velocimetry (PIV).

The experimental setup is presented in Sect. 2 and comprises details of the experimental facility and model (Sect.2.1), the PIV setup (Sect. 2.2), and the investigated test cases (Sect.2.3). The experimental results of this study are presented and discussed in Sect. 3-subdivided into a discussion of the incoming boundary layer and its sensitivity to experimental conditions (Sect. 3.1), the overall flow topology (Sect. 3.2) and the turbulence behavior (3.3).

\section{Experimental facility and setup}

\subsection{Wind tunnel, jet-simulation facility, and experimental model}

Experimental investigations were performed in the hypersonic Ludwieg tube Braunschweig (HLB) in its supersonic configuration (see Fig. 1). In this setup, the original Mach 6 tunnel is modified with a tandem-nozzle configuration including a Mach 2.9 Laval nozzle and an additional settling chamber to create a supersonic wind tunnel with effective measurement times of approximately $60 \mathrm{~ms}$ and a unit Reynolds number range of $\mathrm{Re} / m=1.2 \cdot 10^{6}-17.6 \cdot 10^{6}$. For details on this configuration of the HLB facility, see Wu et al. [13].

The experimental conditions for our present study were chosen to agree with the supersonic test case of the collaborative research center SFB TR40, corresponding to a space-launcher trajectory point at an altitude of $25 \mathrm{~km}$, and are summarized in Table 1.

The wind-tunnel model, a generic representation of the Ariane 5 launcher's main stage with the Vulcain 2 engine, consists of a nose cone and two cylindrical bodies representing the main body and nozzle fairing, respectively. The geometry and dimensions of this generic model of a space launcher are shown in Fig. 2. The model is installed in the wind tunnel with a sword-shaped strut support, fixed in a

Table 1 Experimental conditions: freestream Mach number $M_{\infty}$, mean freestream velocity $U_{\infty}$ in the streamwise direction, Reynolds number $\operatorname{Re}_{D}$ based on the model diameter $\mathrm{D}$, pressure $p_{\mathrm{SP}}$ in the storage tube, and temperature $T_{\mathrm{RK}}$ and pressure $p_{\mathrm{RK}}$ in the settling chamber

\begin{tabular}{llllll}
\hline$M_{\infty}$ & $U_{\infty}$ & $\operatorname{Re}_{D}$ & $p_{\mathrm{SP}}$ & $T_{\mathrm{RK}}$ & $p_{\mathrm{RK}}$ \\
\hline 2.9 & $607 \mathrm{~m} / \mathrm{s}$ & $1.3 \cdot 10^{6}$ & $20 \mathrm{bar} \pm 0.25 \%$ & $285 \mathrm{~K} \pm 3.5 \%$ & $1.52 \mathrm{bar}$ \\
\hline
\end{tabular}

Fig. 1 Schematic of the hypersonic Ludwieg tube Braunschweig (HLB) in supersonic configuration

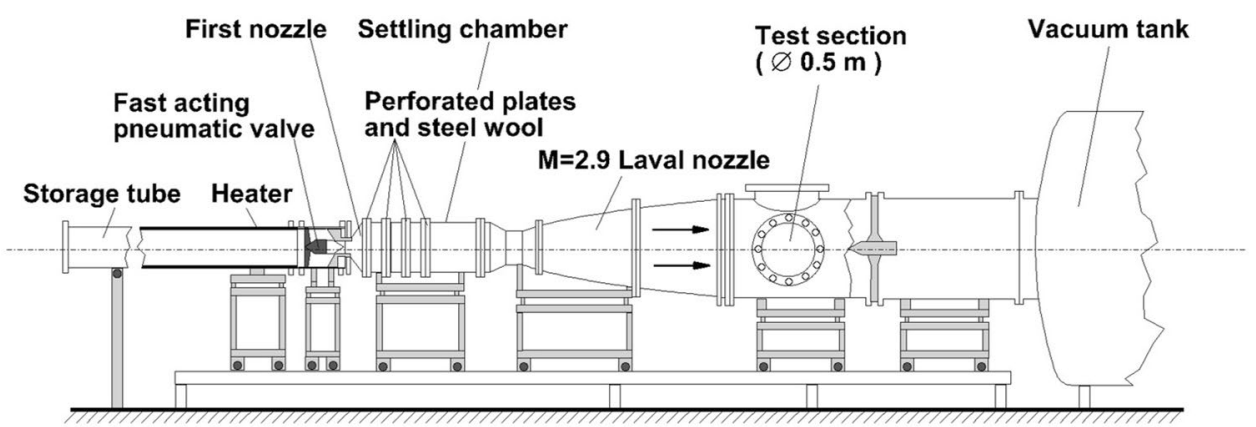




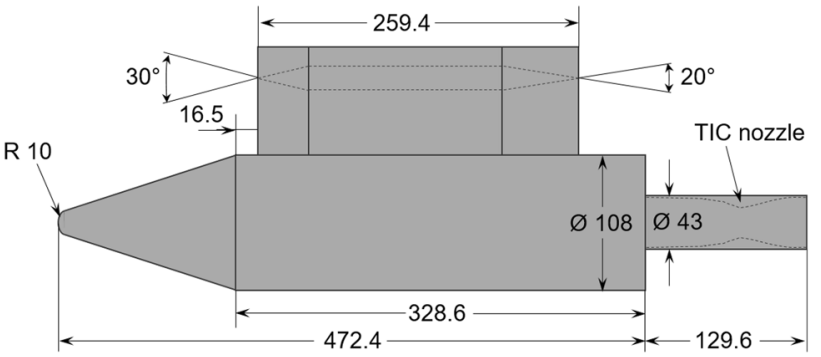

Fig. 2 Sketch of the generic space-launcher model with dimensions in $\mathrm{mm}$

window opening in the upper test-chamber wall. Inlets are fitted into the top and bottom windows (used as a cable outlet) to get a smooth inner surface in the test section and thus minimize disturbances.

To investigate the influence of a propulsive jet on the launcher-wake flow in the HLB facility, the jet-simulation facility (TSA) described by Stephan et al. [7] is integrated in the wind-tunnel model. This facility for the simulation of afterbody flows in the HLB facility works according to the same principle as the HLB itself, with a long heated storage tube that can be pressurized up to 140 bar and heated up to $900 \mathrm{~K}$ outside of the wind tunnel, and a tandem nozzle consisting of two nozzles and an intermediate settling chamber (see Fig. 3a). The second nozzle represents the nozzle of the launcher model and has been designed in the shape of an axisymmetric truncated ideal contour nozzle (TIC) with a mean exit Mach number of 2.5, and a nozzle exit diameter of $d_{\mathrm{e}}=43 \mathrm{~mm}$. The length axis of the launcher model with the TSA is aligned with the HLB center line.

For the present measurement campaign, cold air was used as working gas for the simulation of a moderately underexpanded propulsive jet $\left(p_{\mathrm{e}} / p_{\infty}=5.7\right)$. The jet-simulation parameters are summarized in Table 2. These conditions are neither close to simulating realistic launcher-propulsion conditions, nor is this the aim of this study. I intend to contribute to the qualitative understanding of the influence of an afterexpanding propulsive jet on the wake flow, as well as to create a validation data set.

Particle image velocimetry (PIV) measurements were performed in a vertical plane on the opposite side of the strut $\left(\theta=180^{\circ}\right.$, bottom side of the launcher model) to reduce influences of the flow disturbances induced by the support as much as possible. Schlieren visualizations of the flow field were carried out in the same plane. Schlieren images were taken in a single-mirror coincident setup, using a mercury vapor lamp as light source. A sequence of images with an exposure time of $1.5 \mu \mathrm{s}$ was recorded with a Phantom v711 high-speed camera at a recording frequency of 22,006 fps. Three Kulite XCS-093 pressure sensors with a pressure range of 0.35 bar absolute were flush mounted into the main

Table 2 Jet-simulation parameters: nozzle exit Mach number $M_{\mathrm{e}}$, jet total pressure $p_{t, S C}$, total temperature $T_{t, e}$ at nozzle exit, nozzle to freestream pressure ratio $p_{\mathrm{e}} / p_{\infty}$, and freestream to maximum velocity ratio $\left(u_{\max }-u_{\infty}\right) / u_{\max }$ with $u_{\max }=\left[(2 \kappa) /(\kappa-1)\left(\Re \cdot T_{t}\right) / M_{\mathrm{Mol}}\right]^{-0.5}$

\begin{tabular}{lllll}
\hline$M_{\mathrm{e}}$ & $p_{t, S C}$ & $T_{t, e}$ & $p_{\mathrm{e}} / p_{\infty}$ & $\left(u_{\max }-u_{\infty}\right) / u_{\max }$ \\
\hline 2.5 & $4.1 \mathrm{bar}$ & $280 \mathrm{~K}$ & 5.7 & 0.2 \\
\hline
\end{tabular}

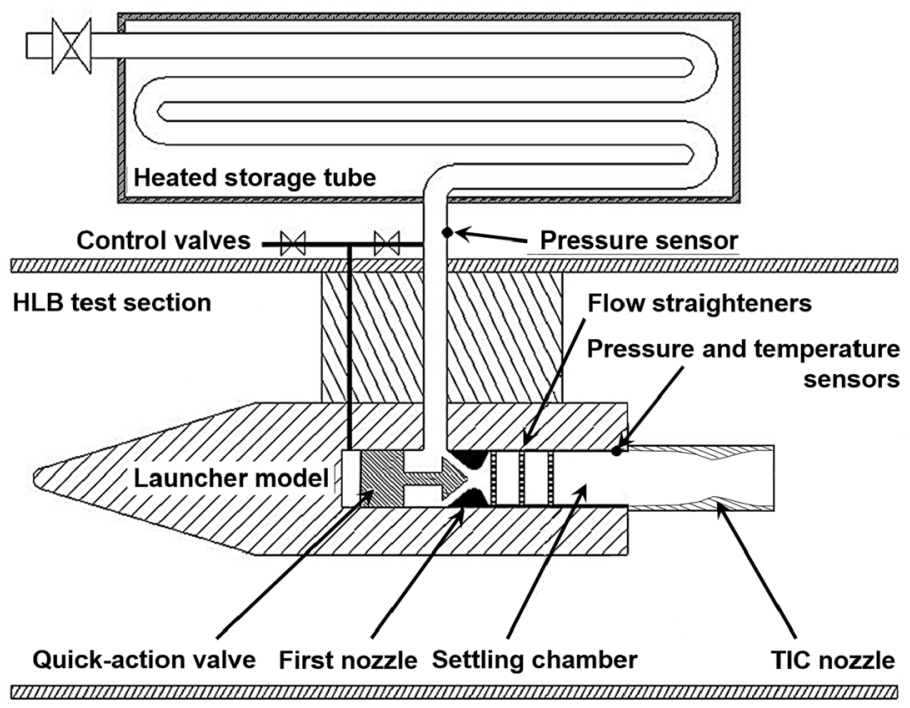

(a)

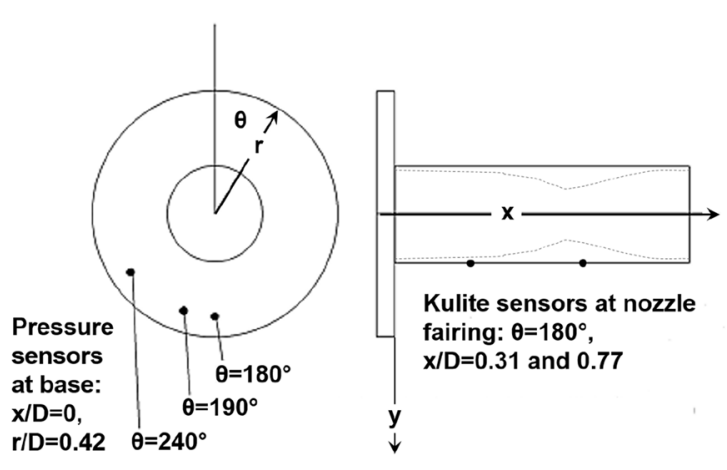

(b)

Fig. 3 Schematic of the jet-simulation facility (a) and locations of pressure sensors (b) 
body base at a radial position of $r / D=0.42$ and at different angles $\left(\theta=180^{\circ}, 190^{\circ}\right.$, and $\left.240^{\circ}\right)$ to capture variations in the azimuthal direction. Two additional sensors were placed at $x / D=0.31$ and $x / D=0.77\left(\theta=180^{\circ}\right)$ on the nozzle fairing (see Fig. 3b). The measurement locations were chosen based on previous studies on the same model geometry [7, 9]. A Spectrum M2i.4652 transient recorder was used to sample the pressure signals. Fluctuations with frequencies below $200 \mathrm{~Hz}$ and above $50 \mathrm{kHz}$ (the cut-off frequency of the sensors) were filtered out of the signal.

\subsection{Particle image velocimetry setup}

A Litron Nano T180-15 PIV double-pulse Nd-YAG laser with a pulse energy of $150 \mathrm{~mJ}$ was used to illuminate the area of interest, namely the wake-flow region. The lightsheet had a thickness of $1 \mathrm{~mm}$. The field of view (FoV) was illuminated from the downstream direction to minimize reflections. The laser optical setup was placed downstream of the vacuum tank, which is equipped with a quartz glass window.

Due to the long optical path, special care was taken in the process of aligning the laser sheet with the intended measurement plane and location of the field of view.

Two LaVision Imager Pro X $11 \mathrm{M}$ cameras with $4008 \times 2672$ pixels CCD chips and Tamron SP AF $180 \mathrm{~mm}$ F3.5 macro lenses were used to record the PIV images. The cameras were mounted on a stall built around the wind-tunnel test section, decoupled from the wind-tunnel vibrations.

Oil droplets of a temperature-resistant lubricant oil (Plantfluid) were used to seed the outer flow, yielding a mean droplet size just below $200 \mathrm{~nm}$. The seeding droplets were introduced into the wind-tunnel storage tube prior to each wind-tunnel run. Note that the jet flow was not seeded in this study. The PIV system has been discussed in detail by Casper et al. [14].

\subsection{Investigated test cases and conditions}

In the PIV measurement campaign, both the basic wake flow and the influence of a propulsive jet on the structure of the flow field were investigated.
The area of interest for both cases was the region along the nozzle outer surface downstream of the launcher model base (see Fig. 4), covering the development region of the shear layer along with the separation and reattachment of the flow on the afterbody.

The spatial resolution in the PIV recordings was chosen in such a way that the turbulent quantities could be resolved. To keep errors due to peak locking to a minimum, a sufficiently large average particle displacement was chosen. The conditions for the PIV measurements were determined by applying the model of Angele and Muhammad-Klingmann [15], which states that the discretization velocity $u_{d}$ should be $u_{d} \leq 2 \cdot u_{\mathrm{rms}}$ to reduce the peak-locking error in the corresponding velocity rms fluctuations $u_{\mathrm{rms}}$ to approximately $1 \%$. Based on a preliminary numerical study, I estimated the turbulence intensity to approximately $u_{\mathrm{rms}}=2-3 \% U_{\infty}$.

The required conditions can be achieved by adjusting the temporal delay between the two frames, as well as the spatial resolution in the recordings, which decreases the size of the field of view (FoV) to a fraction of the area of interest. Since I still wanted to be able to observe as much of the near wake-flow field as possible, two cameras of the same type were used, and the area of interest was split up into two fields of view (FoVs A and B, see Fig. 4). Considerable care was taken to obtain the same recording conditions in both FoV. The resulting conditions are summarized in Table 3.

Images were analyzed with LaVisions $\mathrm{DaVis} 7.2$ software, using minimum interrogation windows of $32 \times 32$ pixels $\left(=0.63 \times 0.63 \mathrm{~mm}^{2}\right)$ with $50 \%$ overlap. Only image pairs with at least $80 \%$ valid vectors were considered for further analysis.

Table 3 PIV recording conditions: calibration $\delta x$, maximum streamwise particle displacement $\Delta x_{\max }$, temporal delay between two images $\Delta \tau$, discretisation velocity $u_{d}$, and resulting size of the field of view

\begin{tabular}{lllll}
\hline$\delta x$ & $\Delta x_{\max }$ & $\Delta \tau$ & $u_{d}$ & FoV \\
\hline 50 pix $/ \mathrm{mm}$ & 25 pixels & $0.82 \mu \mathrm{s}$ & $24.4 \mathrm{~m} / \mathrm{s}$ & $80 \times 53 \mathrm{~mm}^{2} \equiv 0.74 \mathrm{D} \times 0.5 D$ \\
\hline
\end{tabular}

Fig. 4 a Locations and sizes of the FoV in the PIV measurement campaign in respect to the generic launcher model. b Definition of the local coordinate system

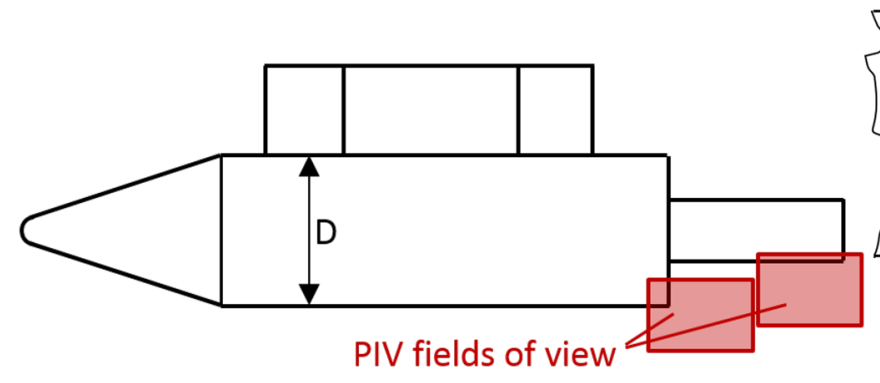

(a)

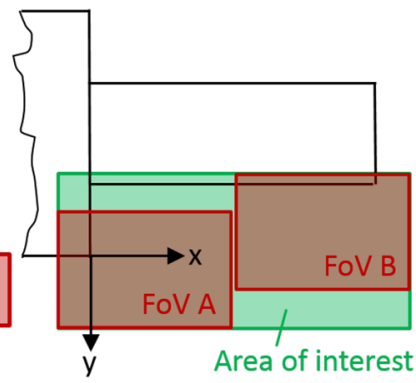

(b) 


\section{Results and discussion}

\subsection{Incoming boundary layer and PIV sensitivity to experimental conditions}

Prior to the analysis of the flow physics, the influence of variations in the nominally stable experimental conditions and of sample size will be discussed. Due to the functional principle of the wind-tunnel facility, the experimental effort for PIV measurements is high, restricting the achievable sample sizes to 400 image pairs per case. It will therefore be assessed whether or not the statistics were sufficiently converged to make reliable statements about the flow. Furthermore, during the measurement campaign, the closing flap in the heating tube of the facility was malfunctioning. Since the flap's functionality is required for measurements at higher temperatures only, and wall-pressure measurements showed excellent repeatability, this was considered negligible. During the analysis of the PIV measurement data, however, it turned out that the flap influenced the turbulence level and slightly even the freestream velocity in the incoming flow. Two different modes of stable flow conditions were obtained in the incoming boundary layer on the main body depending on the orientation of the flap. To attribute the measurement data to the corresponding condition, the PIV data set was split up into subsets of 50 image pairs each and the normalized mean streamwise velocity component $U / U_{\infty}$ along with the rms of the normalized streamwise and radial velocity fluctuation components $u_{\mathrm{rms}} / U_{\infty}$ and $v_{\mathrm{rms}} / U_{\infty}$, respectively, were computed for each of the subsets (not shown here). According to the shape of the $u_{\text {rms }} / U_{\infty}$-profiles, the subsets were categorized into the different modes.

One of the modes clearly represents the mean and turbulent freestream conditions and boundary-layer profiles expected for the chosen operating conditions (see Figs. 5 and 6) and will therefore be discussed in the following paragraphs. For all further data analysis presented in this

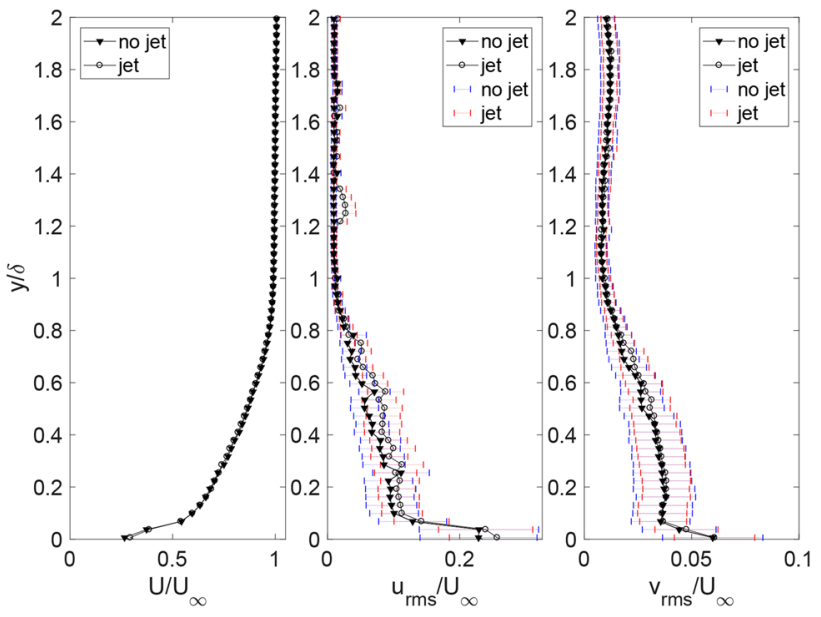

Fig. 6 Mean and turbulent velocity profiles in the boundary layer on the launcher main body with sampling error bars

article, only data sets belonging to this mode were taken into account. For this reason, as well as the fact that only data sets of high quality with at least $80 \%$ valid vectors were considered, the resulting samples of 100 image pairs for the baseline case and 150 image pairs for the propulsive-jet case are of moderate size for statistical PIV analysis. The influence of sample size on the normalized profiles of the mean velocity and the streamwise and radial turbulence intensities across the incoming boundary layer on the launcher main body is shown in Fig. 5 a-c, respectively, for the propulsive-jet case. The rms profiles are not yet smooth and a better convergence can be expected for larger sample sizes. However, for sample sizes from 60 to 150 image pairs, no modifications in trend or magnitude can be observed in the analyzed quantities. I therefore conclude that the topology of the flow field and the influence of a propulsive jet on the topology can be analyzed and discussed, at least in a qualitative manner, based on this data set.

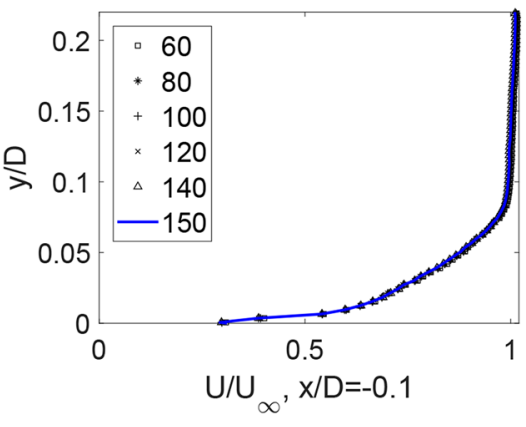

(a)

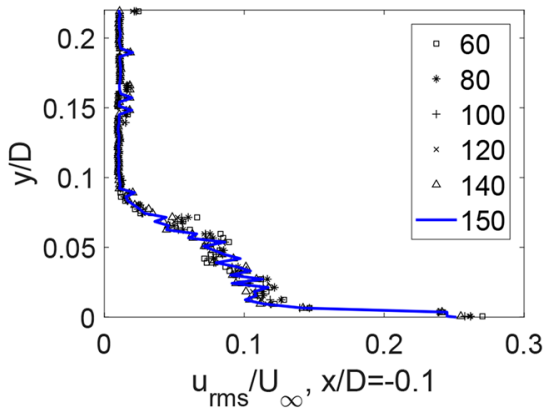

(b)

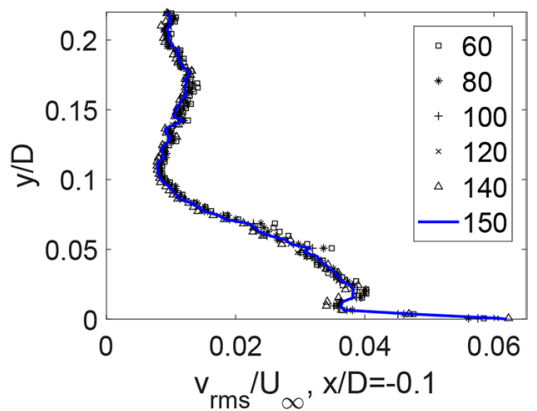

(c)

Fig. 5 Influence of sample size for the propulsive-jet case 
For the investigated cases without and with propulsive jet, velocity profiles in the incoming boundary layer, extracted at $x / D=-0.1$ on the launcher main body and normalized with $U_{\infty}$, are shown in Fig. 6 (left). The boundary layer on the launcher main body $(x / D<0)$ was fully turbulent in both cases. The normalized rms profiles $u_{\mathrm{rms}} / U_{\infty}$ and $v_{\mathrm{rms}} / U_{\infty}$ of the turbulent velocity components in the streamwise and radial directions are shown in Fig. 6 (center) and (right), respectively. The profile shapes are typical for turbulent boundary layers. The sampling uncertainty of the velocity variances across the boundary layer was estimated based on a $95 \%$ confidence interval. The corresponding error bars for each distance from the wall are shown in Fig. 6 (center) and (right).

\subsection{Mean-flow topology}

To provide an overview of the organization of the mean flow field, schlieren optic visualizations covering the entire area of interest marked in Fig. 4 are presented in the bottom and top images in Fig. 7 for the cases with and without propulsive jet, respectively. The presented images were averaged from 400 snapshots each. Note that the images are rotated compared with Figs. 2 and 4, such that the $y$-axis is positive in the upward direction.

The turbulent boundary layer on the main body separates at the shoulder due to the step decrease in diameter in both cases (origin of the $x-y$-coordinate system marked in Fig. 7 (bottom)). The expansion wave developing from the shoulder can be observed. In the baseline case without jet

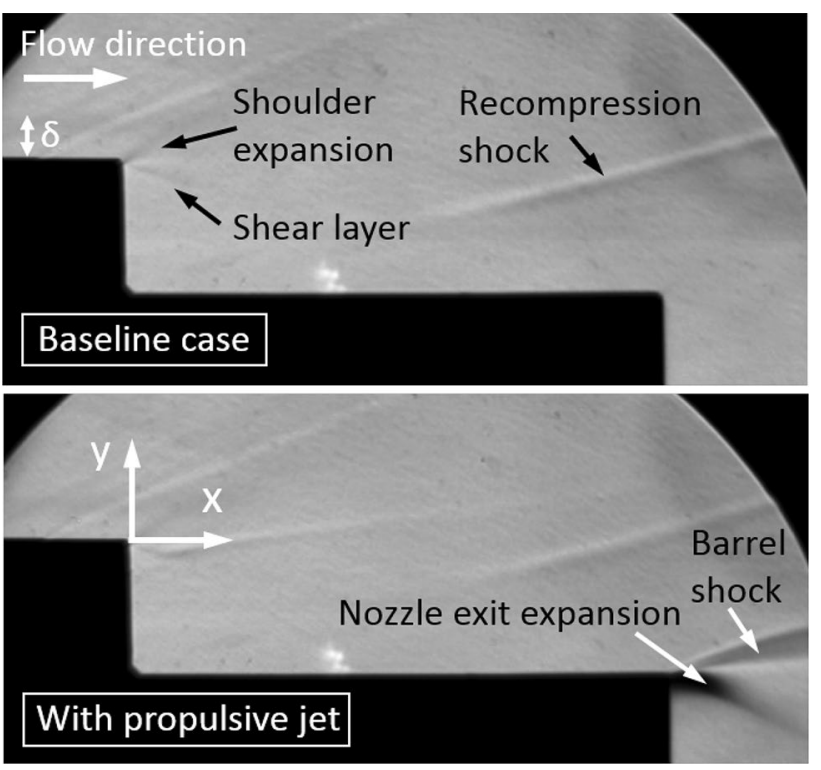

Fig. 7 Schlieren optic visualizations (averaged images) of the afterbody flow. Top image: baseline case, bottom image: propulsive-jet case flow (Fig. 7 (top)), the recompression shock, which indicates the beginning of the reattachment process of the flow on the outer surface of the afterbody [1], is clearly visible. This shock evolves since the shear layer forming downstream of the main body shoulder is bent toward the afterbody and eventually impinges on the surface, which causes another deflection of the flow away from the surface. The most prominent influence of the afterexpanding jet plume on the flow organization, apart from the barrel shock forming downstream of the nozzle exit and the jet-expansion fan, can be observed from the behavior of the reattachment shock. In the jet case (Fig. 7 (bottom)), it is located slightly farther away from the nozzle surface (at $y / h=0.69$ instead of $y / h=0.56$, where $h$ is the height of the step between main body and afterbody) and also farther downstream (at $x / h=2.69$ instead of $x / h=2.33$ ) compared with the baseline case. This visualizes the displacement effect of the jet plume on the outer flow (compare the two images in Fig. 7). Furthermore, the shock angle increases slightly from $14^{\circ}$ to $15^{\circ}$ and the shock only develops fully farther away from the surface. This indicates that the flow does not fully reattach in this case, although the shear layer is still deflected toward the surface and a gradual but incomplete deflection away from the surface creates Mach waves that coalesce into a recompression shock farther downstream.

To take a closer look at the flow topology, the mean velocity fields $U$ in the axial x-direction from PIV (normalized with the mean incoming flow velocity $U_{\infty}$ ) are shown in Fig. 8 a, b for the baseline and jet cases, respectively. Streamtraces are superimposed (in white) onto the velocity contours.

Starting from the shoulder of the launcher main body $(x / D=0)$, a shear layer starts to develop and a large separation zone forms in the corner. Under the influence of the propulsive jet, this separated zone becomes more prominent and increases in size (compare Fig. 8a, b).

From the streamtraces in Fig. 8, as well as the contours of the normalized mean velocity $V / U_{\infty}$ in the radial direction shown in Fig. 9, it is clearly visible that the flow is deflected toward the launcher afterbody, confirming the observations made on the schlieren images.

In the baseline case, the flow starts to reattach onto the outer surface of the afterbody around the streamwise location $x / D \approx 0.75$, as visible in Fig. 10 , where the streamwise mean velocity profiles along the nozzle fairing $(0.6 \leq x / D \leq 1.2)$ for both the baseline and propulsive-jet cases are shown to visualize the reattachment process. For $x / D<0.75$, the shape of the profile with inflection points and displacement from the wall indicates separation. This inflection point moves closer to the wall toward reattachment. Negative velocities close to the wall are only visible to a small extent in the velocity profiles from PIV, since the seeding close to the wall and especially in recirculation 
Fig. 8 Contours of the normalized mean velocity component $U / U_{\infty}$ in the axial main flow direction from PIV measurements. a Baseline case, $\mathbf{b}$ propulsive-jet case

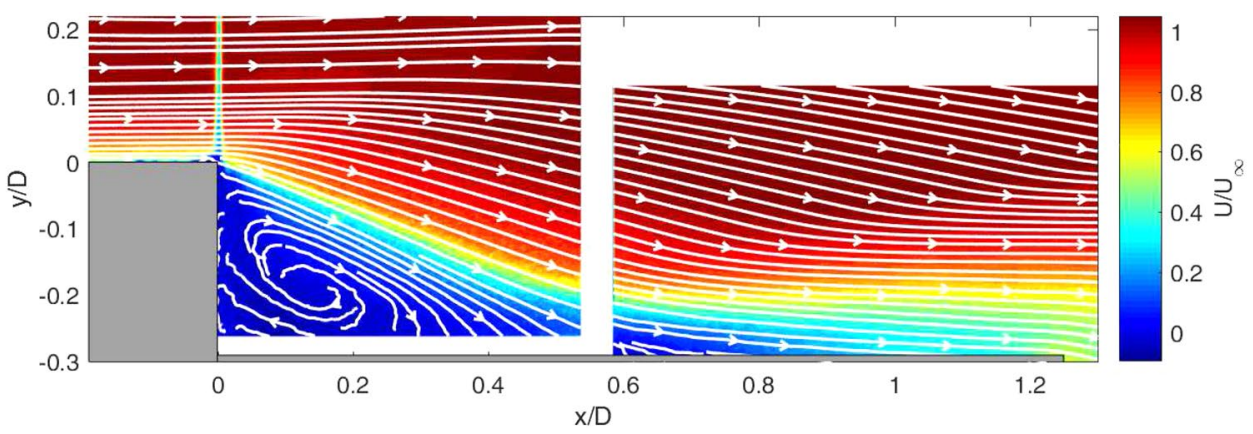

(a)

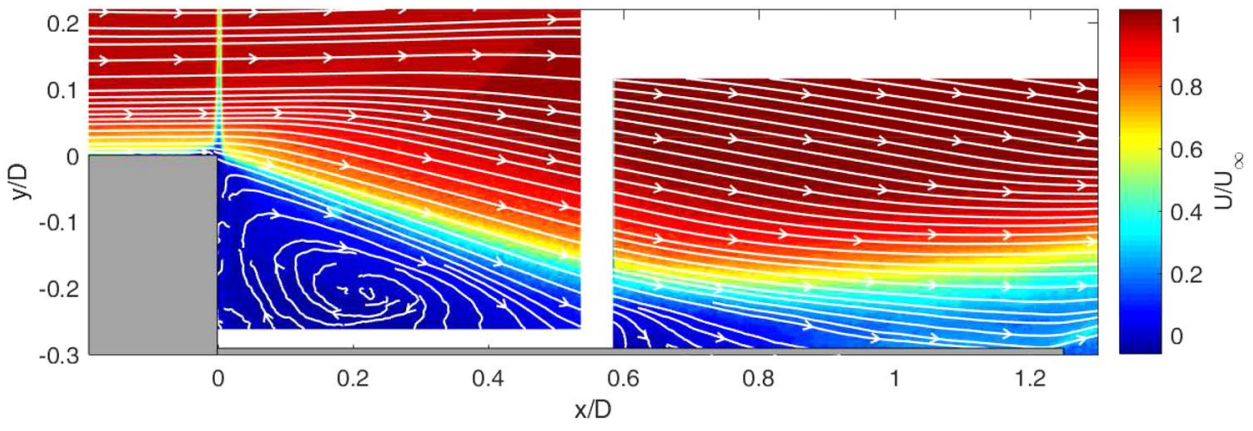

(b)

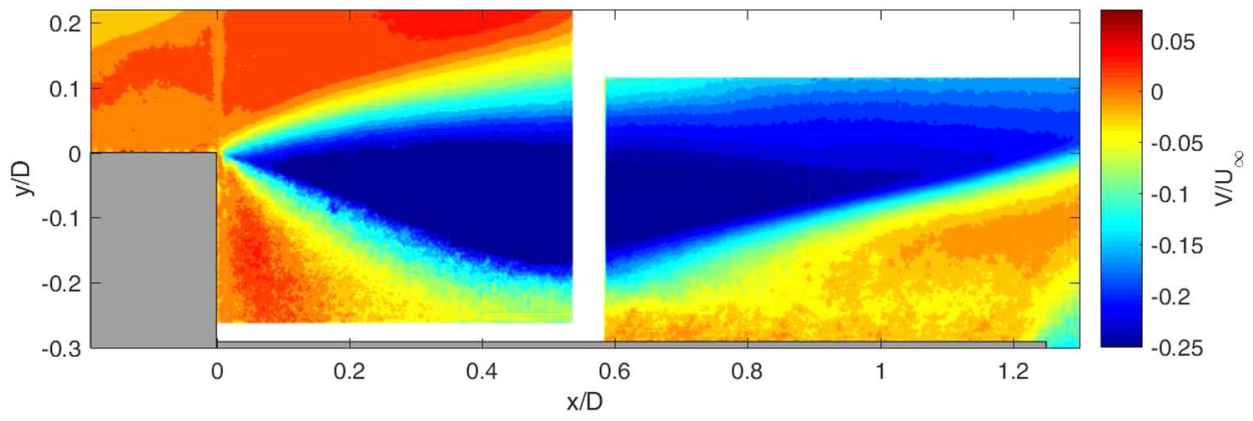

(a)

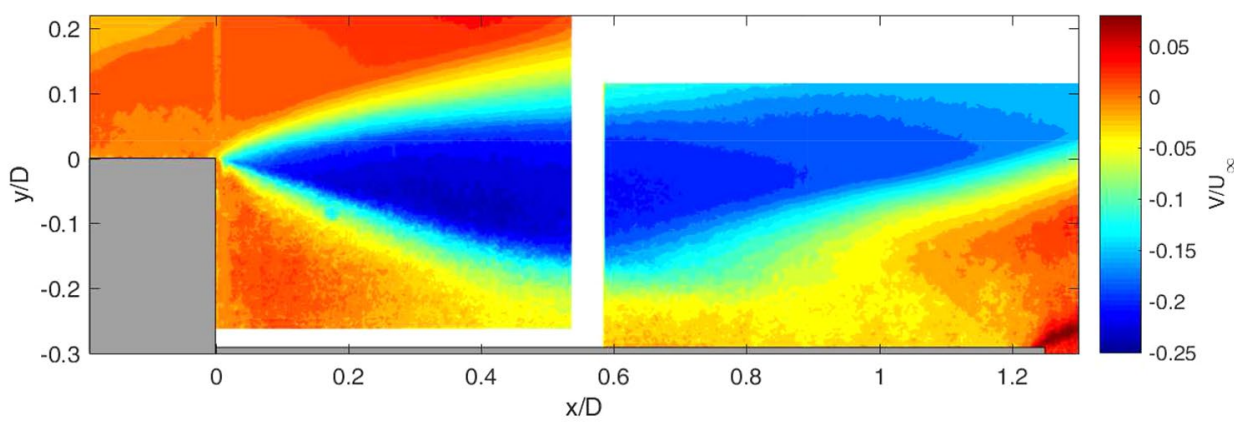

(b) zones is exceedingly difficult. From $x / D=0.75$, another inflection point in the profile appears close to the wall, indicating the start of the reattachment process. With increasing downstream distance, the flow starts to relax and an increasing part of the profile in the wall-near region starts to return to the shape typical for a turbulent boundary layer. Due to the small length of the afterbody, the boundary layer does not reach equilibrium conditions. Under the influence 
Fig. 10 Normalized mean streamwise velocity profiles in the vicinity of the reattachment zone

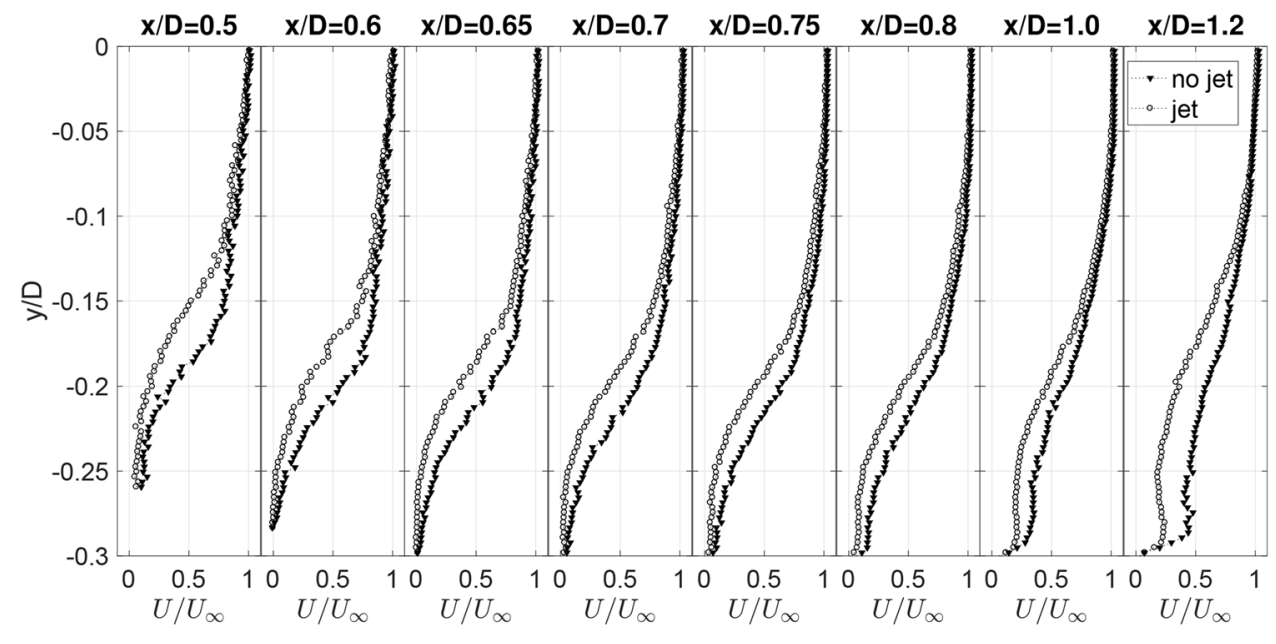

of the propulsive jet, the first indication for reattachment is visible at $x / D=0.8$. This only affects the inner region of the boundary layer $(\leq 0.14 \delta$ at $x / D=1.0)$, though, and the displacement from the wall remains pronounced and always larger than in the baseline case. The reattachment process is not completed along the length of the nozzle fairing. From $x / D=1.2$, the strong displacement by the jet plume and the effect of the expansion at the nozzle exit become increasingly visible by a decrease in mean velocity, e.g., compared with the profile at $x / D=1.0$. These observations agree with the strength and location of the reattachment shock in the schlieren visualizations shown in Fig. 7.

The displacement effect of the jet plume can also be seen in the $U / U_{\infty}$-contours (see Fig. 8), and even more clearly in the $V / U_{\infty}$-contours shown in Fig. 9. Also, the resulting deflection toward the wall is weaker in the wake under the influence of the jet plume (compare the sizes and magnitudes of the zones of negative radial velocity in Fig. 9a, b, respectively).

The direct influence of the jet plume can also be seen in the $V / U_{\infty}$-contours for locations $x / D>1.1$ in form of positive velocities in the radial direction (Fig. 9).

From the normalized mean velocity fields $V / U_{\infty}$ in the radial y-direction shown in Fig. 9, it can be verified that the wall-normal component was indeed zero in the incoming flow as expected.

\subsection{Turbulence behavior in the flow field}

The turbulence behavior, especially the development of the shear layer starting to form at the main body shoulder, will be discussed based on the normalized turbulent fluctuations of the velocity components in the axial and radial directions, as well as the Reynolds shear stresses.

The contour plots of the normalized turbulent fluctuation component $v_{\text {rms }} / U_{\infty}$ in the radial direction presented in Fig. 11 give a good overview of the location of the shear layer in both cases of the studied wake-flow field. The deflection toward the wall is clearly visible both in the baseline case and the jet case (Fig. 11a, b, respectively). Up to a streamwise distance of approximately $x / D=0.6$ from the shoulder, the shear layer is distinctly recognizable from the local maximum in turbulence intensity. In the baseline wake, a second, much weaker, maximum farther away from the surface starts to develop around location $x / D=0.7$ at $y / D \approx-0.11$, which indicates the formation and location of the reattachment shock (see Fig. 11a). In general, the extent of the shear layer in radial direction (i.e., the width of the zone of maximum turbulence intensity) is larger for the case without propulsive jet.

Another feature that immediately catches the eye is the difference in turbulent intensity between the two investigated cases. The overall intensity level is lower in the flow field influenced by the propulsive jet, and the maximum values are lower as in the baseline case as well. It appears that the propulsive jet moderates the turbulence development in the wake-flow field.

For a more detailed analysis, profiles of rms values of the fluctuating velocity components were extracted for several locations between $0.05 \leq x / D \leq 1.2$ along the afterbody. For both investigated cases, the respective axial components are shown in Fig. 12 and the radial components in Fig. 13. Both quantities are normalized with $U_{\infty}$. In both the axial and radial components, a local intensity maximum in the profile can be observed, indicating the location of the shear layer (e.g., at $y / D=-0.02$ at $x / D=0.005$ for $u_{\text {rms }} / U_{\infty}$ ). Up to the reattachment region, the shear layer and thus the location of the maximum shifts toward the nozzle fairing with streamwise distance from the shoulder. Its deflection away from the wall after impinging on the nozzle fairing, especially in the propulsive-jet case, is visible in both components. Also the previously discussed displacement effect of the jet plume on the outer flow can be observed clearly when comparing the respective profiles for the baseline and propulsive-jet cases 
Fig. 11 Contours of the normalized turbulent fluctuation component $v_{\text {rms }} / U_{\infty}$ in the radial direction from PIV measurements. a Baseline case, b propulsive-jet case

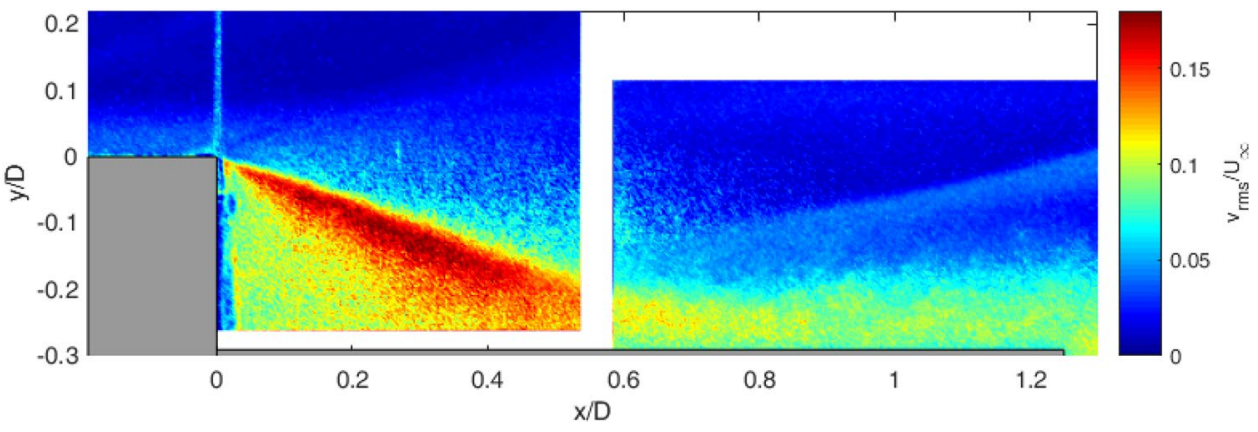

(a)

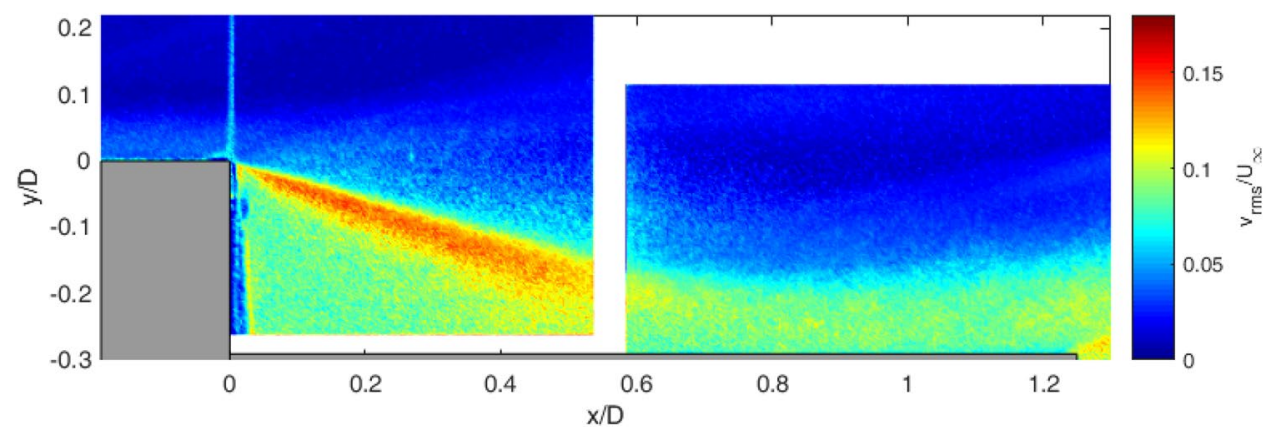

(b)

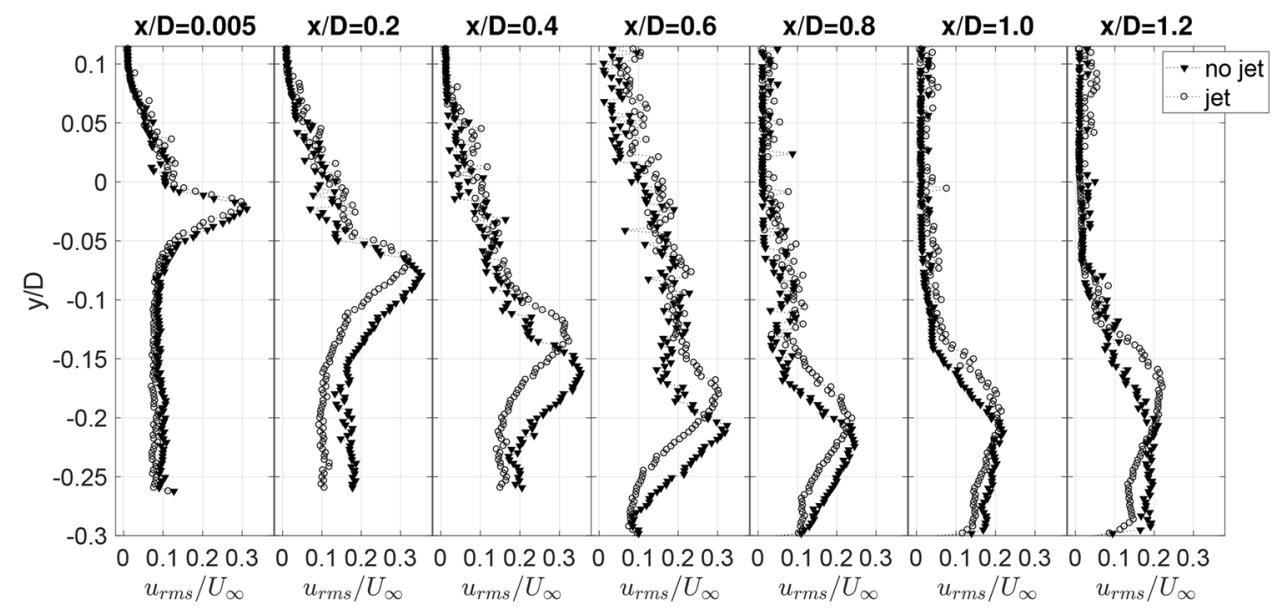

Fig. 12 Profiles of the turbulent fluctuations $u_{\mathrm{rms}}$ of the axial velocity component normalized with the mean incoming flow velocity $U_{\infty}$ along the launcher afterbody in Figs. 12 and 13: the location of the shear layer (indicated by the local maximum in the turbulence intensity profile) is farther away from the surface under the influence of the jet plume for corresponding streamwise locations.

In both fluctuating velocity components, the respective maximum values are higher for the baseline case up to $x / D=0.8$. At $x / D=0.2$, for example, $v_{\text {rms }} / U_{\infty}$ reaches a turbulent intensity of $17.2 \%$ in the baseline case, compared to $13.5 \%$ in the case influenced by the jet plume. For $u_{\text {rms }} / U_{\infty}$, the effect is smaller, but can still be observed (see Figs. 12 and 13, respectively).

In the baseline case (see Fig. 13), the maximum of the radial component of the turbulent velocity fluctuations increases continuously until a distance from the base of $x / D=0.4$. This is expected for shear-layer instability. At the same time, the shear layer moves from the shoulder toward the outer surface of the nozzle fairing, then interacts with the wall and the boundary layer, and is finally reflected. The maximum representing the shear layer starts to decrease and then vanishes. From a streamwise distance of $x / D=0.8$ onward, the distribution of the local intensity maximum close to the wall $(y / D \approx-0.25)$ approaches that of a disturbed turbulent boundary layer that is starting to relax. Equilibrium conditions cannot be reached until the nozzle exit due to the small length of the afterbody. 
Fig. 13 Profiles of the turbulent fluctuations $v_{\text {rms }}$ of the radial velocity component normalized with the mean incoming flow velocity $U_{\infty}$ along the launcher afterbody

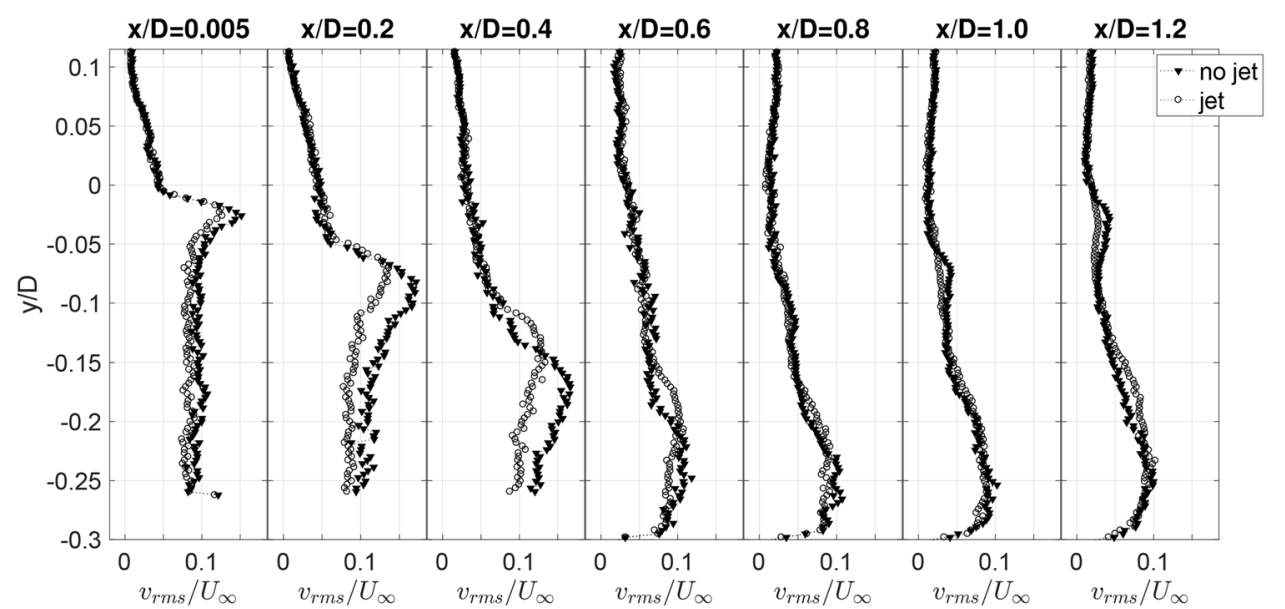

Farther away from the wall, a second intensity maximum develops from $x / D \geq 0.8$ and shifts away from the wall with increasing streamwise distance. This represents the reattachment shock.

Under the influence of the jet plume (see Fig. 13), the increase of turbulent intensity in the developing shear layer seems damped (no continuous increase can be observed). The outer maximum corresponding to a reattachment shock develops only to a much weaker extent than in the baseline case (see locations $x / D=1.0$ and $x / D=1.2$ in Fig. 13). This again confirms that the reattachment process on the afterbody surface begins, but is not completed in this case.

The same qualitative behavior regarding the shear-layer location and turbulence intensities can be observed in the axial velocity-fluctuation profiles shown in Fig. 12, and even more clearly in the normalized profiles of the Reynolds shear stresses compared in Fig. 14.

Deprés et al. [1] have made a similar observation in a transonic wake flow. They have performed pressure measurements on a number of different afterbody configurations at $M=0.85$, and found that a supersonic propulsive jet interacts with the recirculation region on the nozzle fairing and modifies the flow topology. They observed that the jet stabilizes the near-wake region, leading to a more axisymmetric external flow field [1]. Deprés et al. [1] explained this effect with the physical presence of the propulsive jet in the center of the wake that obstructs the development of large-scale vortical structures.

To further analyze this phenomenon, I will discuss our observations made on surface-pressure fluctuation measurements and analyze the behavior of turbulent structures in the flow field based on a proper orthogonal decomposition (POD) in Sects. 3.4 and 3.5, respectively.

\subsection{Surface-pressure fluctuations}

Some information on the dynamic wake flow behavior can be gained from the power spectral densities (PSD) of the wall-pressure fluctuations measured at the base of the launcher-model main body and along the nozzle fairing, since the pressure signal represents a footprint of the turbulent structures in the wake [1]. For the two
Fig. 14 Profiles of the Reynolds shear stresses $\overline{u^{\prime} v^{\prime}}$ normalized with the mean incoming flow velocity $U_{\infty}^{2}$ for several locations along the launcher afterbody

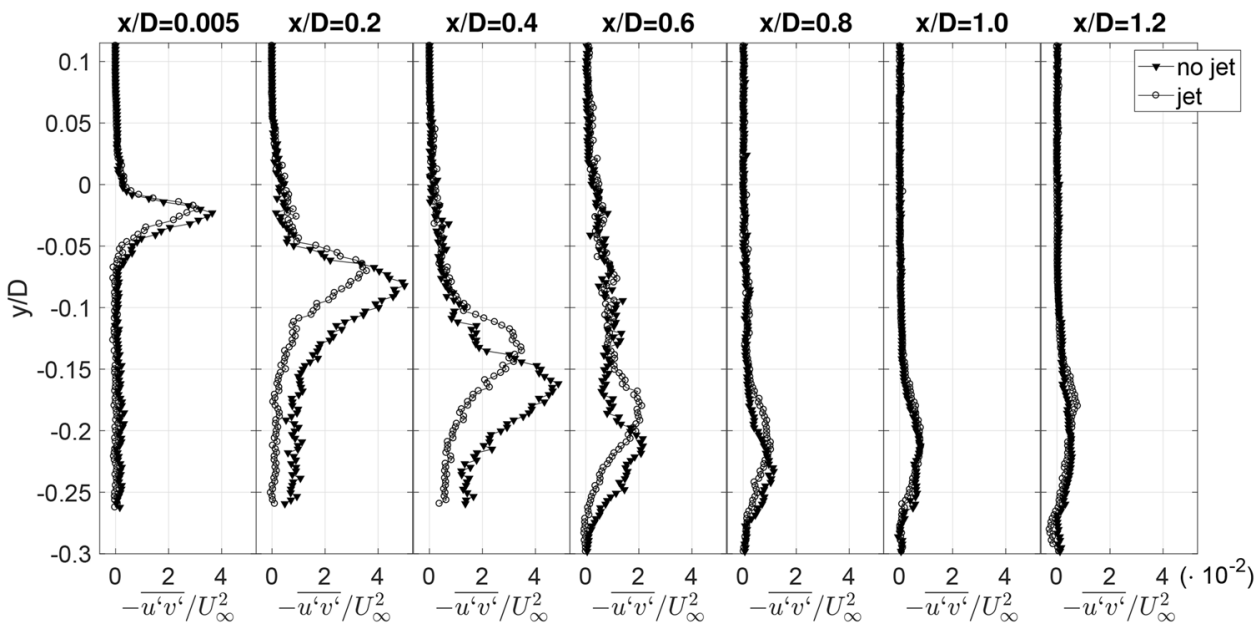


measurement zones, the PSD (in $P a^{2}$ ) is plotted versus the Strouhal number $S t_{D}$ based on main body diameter $\mathrm{D}$, i.e., $S t_{D}=f \cdot D / U_{\infty}$ in Fig. $15 \mathrm{a}$, b, respectively, for both investigated cases. As mentioned previously, the pressure sensors were located at a radius of $r / D=0.42$ at three different angular positions $\left(\theta=180^{\circ}, 190^{\circ}\right.$, and $\left.240^{\circ}\right)$ on the main body base, and two locations, $x / D=0.31$ and $x / D=0.77$ at $\theta=180^{\circ}$, along the nozzle fairing (see Fig. 3b). The PSD was computed with Welch's method based on time traces of $30 \mathrm{~ms}$. For segmentation, Hamming windows of 90,000 points were used at $50 \%$ overlap. The PSD are shown averaged from 38 readings for each sensor for both the baseline (bold lines) and the jet cases (thin lines), respectively.

The behavior of the intensity peak for $\mathrm{St}_{D}=0.21$ that can be observed in the baseline case is of specific interest here. This peak has previously been observed in different configurations over a large range of Mach numbers [1, $5,11,16,17]$, and is generally attributed to the vortex shedding from the separation bubble. It is governed by the interaction between those turbulent structures and the wall in the reattachment zone [1]. The peak disappears in azimuthal direction (see Fig. 15a), which is related to helical vortex structures, as Deprés et al. [1] and Deck and Thorigny [2] have discussed. These coherent anti-symmetrical fluctuations are relevant for uneven side loads and the buffet problem.

In the propulsive-jet case, this peak is more broadband, contains less energy, and is shifted to slightly higher frequencies (centered around $S t_{D}=0.26$, see Fig. 15a). The weaker occurrence of this peak in the propulsive-jet case does again back up by the finding that the flow does not fully reattach in this case. The increased Strouhal number indicates that the corresponding turbulent structures are slightly smaller, potentially due to the jet plume inhibiting the growth of the shear-layer structures. In general, the energy content at low frequencies is higher than in the baseline case, but for $\mathrm{St}_{D}>0.3$ the curves follow the same trend, namely a continuous drop in energy content. Note that, due to the strut support used to install the axisymmetric model in the wind-tunnel test section, the flow field is not entirely axisymmetric. This leads, inter alia, to additional energy peaks at higher frequencies $\left(\mathrm{St}_{D}>1.0\right)$ in the propulsive-jet case, which are most likely caused by strong jet noise disturbances that are reflected non-uniformly by the strut support. For a more detailed discussion of the PSD of the base pressure, see Schreyer et al. [18].

On the nozzle fairing, an increase of the energy content at higher frequencies $\left(\mathrm{St}_{D}>0.6\right)$ can be observed in the baseline case at the measurement location $x / D=0.31$ (see Fig. 15b). Farther downstream $(x / D>0.77)$, the energy content increases further and an additional increase in broadband low-frequency content follows. This increased energy content corresponds to the turbulent structures in the shear layer that are transported toward and along the nozzle wall [19]. The energy content at $x / D=0.31$ still exhibits a slight increase around $\mathrm{St}_{D}=0.21$, although a distinct peak cannot be observed. The signal at this location within the separated region thus contains contributions of the interaction of the vortices shed from the separation bubble with the surface. At $x / D=0.77$, no such contribution can be observed anymore. The energy content at this location is generally higher than in the baseline case (except for the $\mathrm{St}_{D}=0.21$ peak) and much more broadband; no sharp drop in energy content can be observed up to $\mathrm{St}_{D}>3$.

A distinct influence of the propulsive jet on the PSD of the pressure signal along the nozzle fairing cannot be observed.

\subsection{Vortical structures in the flow}

The observed stabilizing effect of the jet plume on the wake flow is assumed to be related to the jet obstructing the growth of turbulent structures in the shear layer [1]. Coherent turbulent structures in a shear layer contain large fractions of the turbulent kinetic energy in the flow and can therefore be analyzed based on a proper orthogonal decomposition
Fig. 15 Wall pressure spectra averaged from 38 readings. a At the launcher base, $\mathbf{b}$ along the nozzle fairing

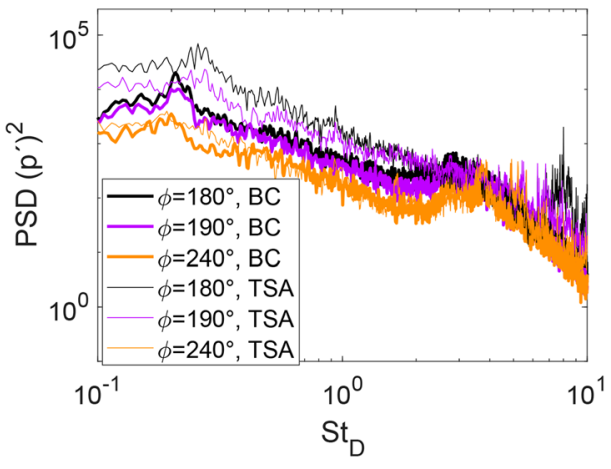

(a) Main-body base

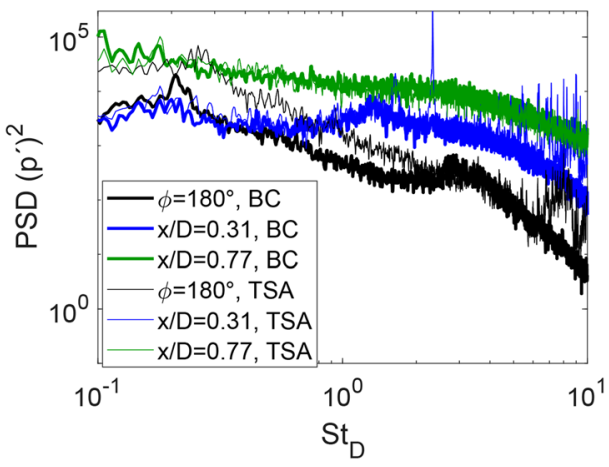

(b) Nozzle fairing 
(POD) of the velocity fields from PIV. POD is a method to extract coherent structures from experimental and numerical data (see Lumley [20] and Berkooz et al. [21]). The resulting spatial modes, corresponding to the spatial representation of the coherent structures, are sorted by descending energy content. For flows such as the shear layer I intend to analyze, already a small number of POD modes are able to capture the relevant energy content and can be used to reconstruct a reduced-order representation of the flow field. Since I applied POD to sets of non-time-resolved velocity fields from PIV measurements, the more suitable snapshot POD (see Sirovich [22]) was applied here. Based on the POD modes resulting from the decomposition, I will analyze the size and energy content of the structures in the wake flow and verify whether or not the propulsive jet limits the growth of the turbulent structures. To make the fractions of energy associated with the respective modes of the same order in the baseline and propulsive-jet cases directly comparable, the POD was applied to 100 snapshots for each of the cases. A preliminary analysis of these cases has been presented by Schreyer [23].

The energy content in the respective eigenmodes $\lambda_{n}$ and the cumulative energy content $\sum_{n}$ after a number of $n$ modes are shown in Fig. 16 for the axial component $\phi_{n, x}$ of the modes. In the eigenspectra for both the baseline and propulsive-jet cases (filled symbols and open symbols, respectively), the first mode contains approximately $10 \%$ of the turbulent kinetic energy. A significant fracture of the kinetic energy is therefore distributed over a larger number of higher-order modes contributing to the dynamic behavior of the wake flow.

The first five eigenmodes $\phi_{1, x}-\phi_{5, x}$, representing normalized fluctuations of the axial velocity component, are shown in Fig. 17 for the baseline case (left column) and the propulsive-jet case (right column). The corresponding modes for the radial velocity component $\phi_{1, y}-\phi_{6, y}$ are shown in Fig. 18. The spatial representations of the

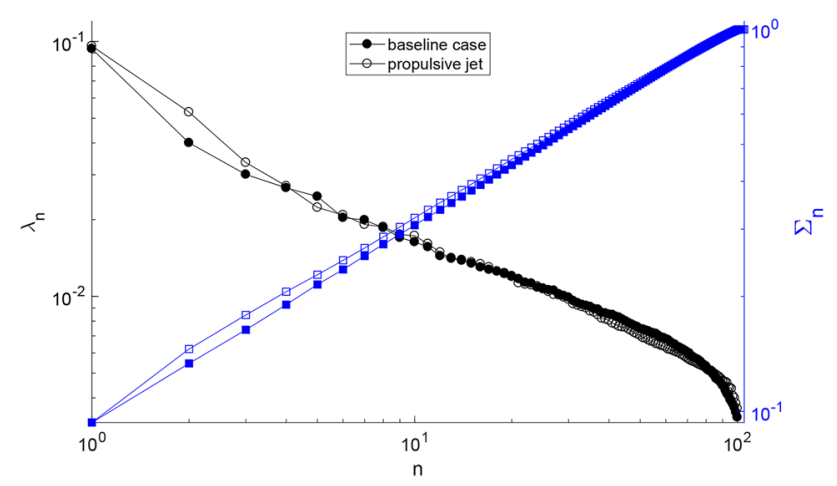

Fig. 16 Eigenspectra $\lambda_{n}$ (circle symbols) and cumulative relative energy $\sum_{n}$ (square symbols) in the first $n$ modes. Filled symbols: baseline case, unfilled symbols: propulsive-jet case presented most energetic modes show similarities in the baseline and propulsive-jet cases, including contributions for the separation and realignment zones, the shear layer, and turbulent coherent structures along the nozzle fairing that are of similar sizes for corresponding modes. Details, and especially the differences between the cases, will be discussed in the following.

The low-frequency shock oscillation typical for highspeed flows with large closed separation regions (see Clemens and Narayanaswamy [24]) can also be observed in the current flow field and a significant part of the turbulent kinetic energy is associated with this phenomenon in the baseline case. Without propulsive jet, the axial and radial components of the first mode have a pronounced energy content of $9.4 \%$ and $6.6 \%$, respectively, in the separation bubble and the reattachment and realignment regions downstream (see Figs. 17a and 18a). The corresponding shock motion is captured by the first radial mode $\phi_{1, y}$ (Fig. 18a), where $6.6 \%$ of the total turbulent kinetic energy are contained in the location of the recompression shock (for $x / D \geq 0.6$ ), the separation and reattachment zones. Also in modes $\phi_{4, y}$ and $\phi_{5, y}$ (Fig. 18g, i, respectively), significant further contributions can be found. Due to the incomplete reattachment process, a much weaker contribution of this phenomenon can be observed in the propulsive-jet case. In the first radial mode $\phi_{1, y}$ (Fig. 18b), containing $4.2 \%$ of the energy, the modal contribution of the reattachment location starts farther downstream and a weak contribution of the coalescing compression waves can be observed for $x / D>0.8$. A further contribution in the same location can be found in the fifth and sixth modes with $1.9 \%$ of the total turbulent kinetic energy each (see Fig. 18j, 1). In the first axial mode $\phi_{1, x}$ for the propulsive-jet case (Fig. 17b), an energy content of 9.6\% can be observed in the separation and realignment zones as well, but also the shear layer already contributes here.

A significant energy contribution is associated with the shear layer forming downstream of the main body shoulder. The axial and radial components of modes two to five partially represent velocity fluctuations in the shear layer in both investigated cases. In the presence of the propulsive jet, this spatial mode extends farther downstream than in the baseline case (see, e.g., mode $\phi_{2, x}$ and $\phi_{2, y}$ in Figs. $17 \mathrm{c}, \mathrm{d}$ and $18 \mathrm{c}$, d). Also the associated energy content is larger, since a corresponding contribution can already be observed in the first, most energetic axial mode $\phi_{1, x}$ in the propulsive-jet case, and the second and third axial modes, $\phi_{2, x}$ and $\phi_{3, x}$, respectively, also contain larger fractions of energy than in the baseline case $(5.3 \%$ vs. $4.0 \%$ and $3.3 \%$ vs. $3.0 \%$, respectively).

The largest overall contributions to the turbulent kinetic energy of the flow stem from a large number of modes associated with the increasingly small turbulent structures shed from the separation zone and forming in the reshaping boundary layer on the nozzle fairing. In the baseline 


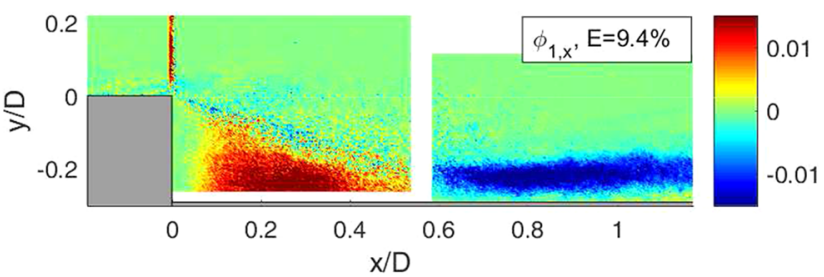

(a)

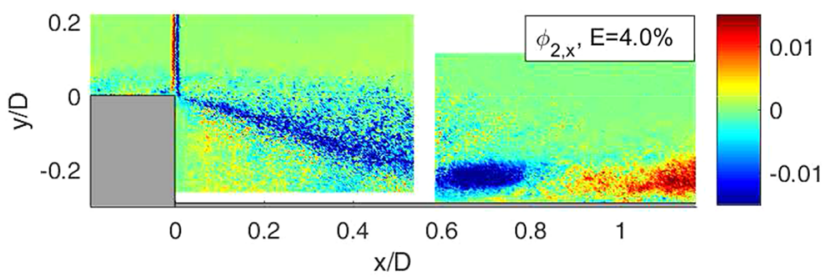

(c)

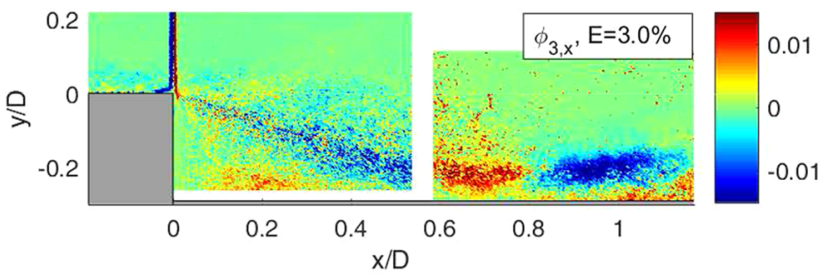

(e)

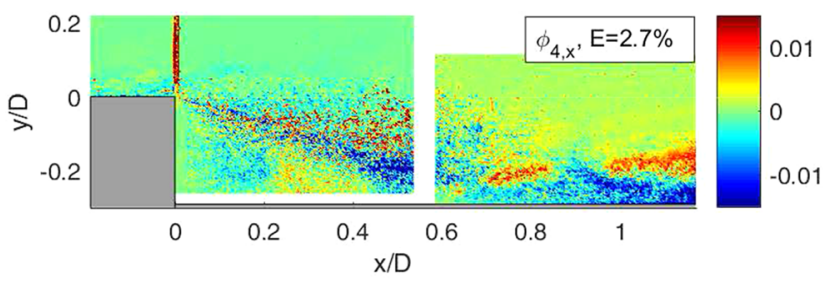

(g)

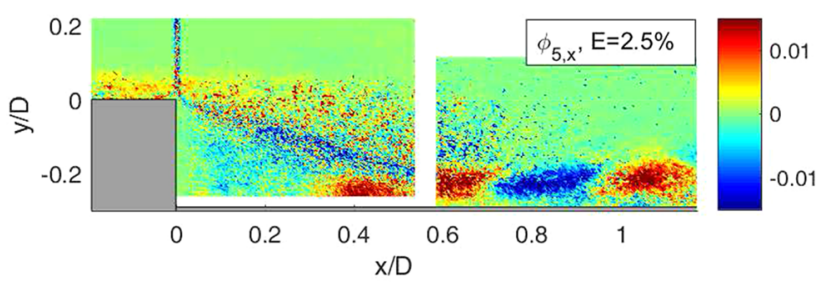

(i)

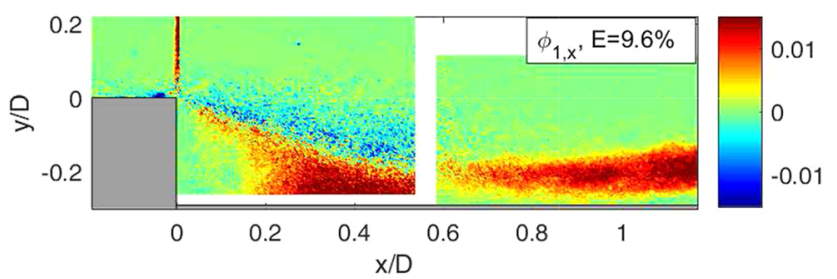

(b)

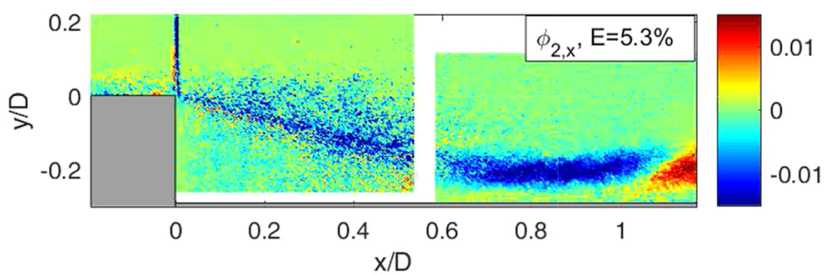

(d)

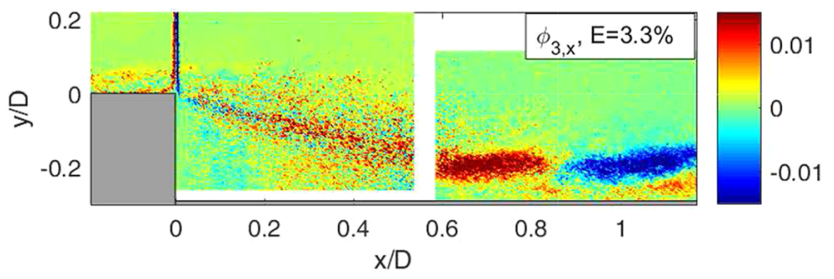

(f)

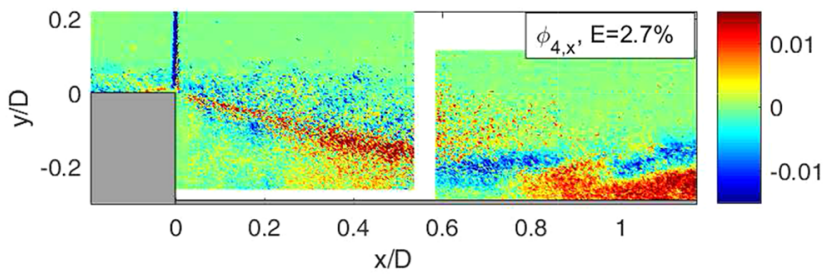

(h)

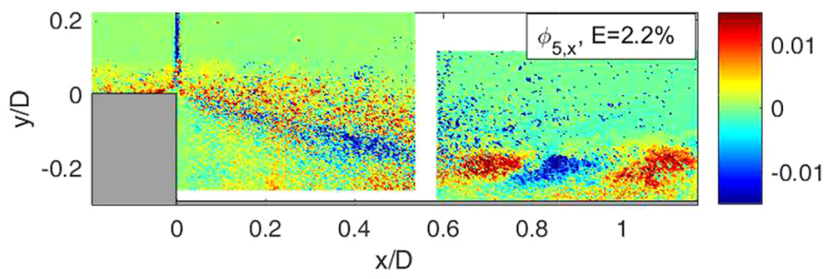

(j)

Fig. 17 POD modes for streamwise velocity fluctuations $\frac{u}{U_{\infty}}$ without (left column) and with propulsive jet (right column)

case, modes three and higher contain $86.6 \%$ and $90.1 \%$ of the energy in the axial and radial components, respectively, and $85.1 \%$ and $92.9 \%$, respectively, under the influence of the propulsive jet. In these higher-order modes, the energy distribution in the two studied cases differs. Modes 4-8, representing larger turbulent structures and modes 20-80 contain a larger fraction of the turbulent kinetic energy in the baseline case than under the influence of the propulsive jet (see Fig. 16). The modes representing very small structures (modes 81-100), on the other hand, accumulate more energy in the propulsive-jet case.

This means that in comparison, in the propulsive-jet case, a larger fraction of the turbulent kinetic energy is contained (a) in motions of the shear layer as a whole 


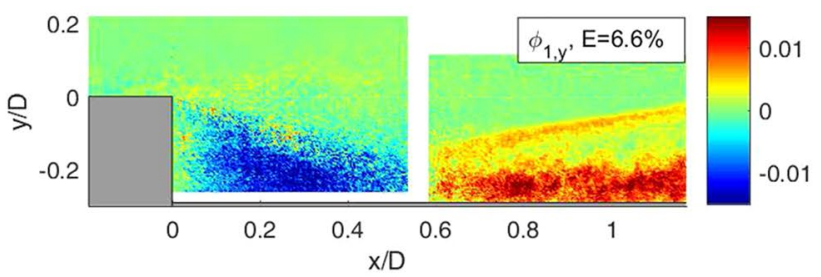

(a)

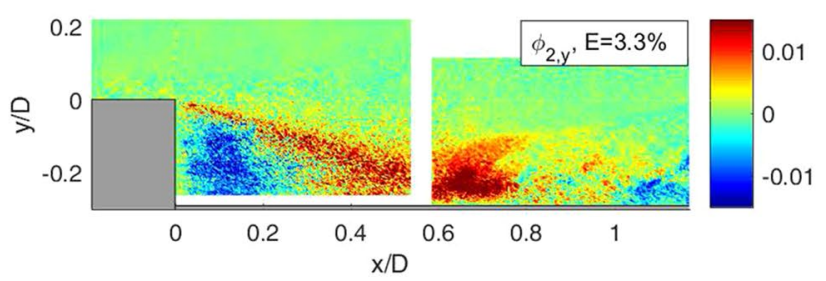

(c)

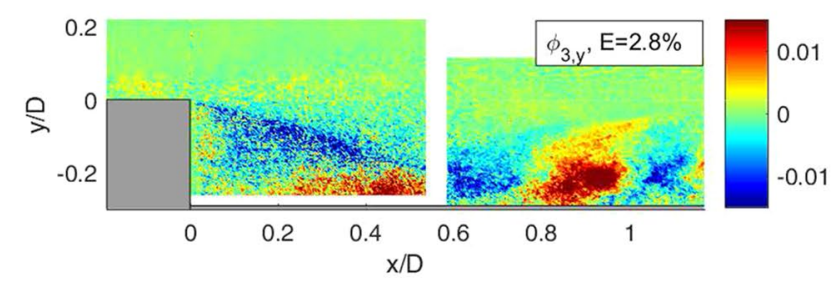

(e)

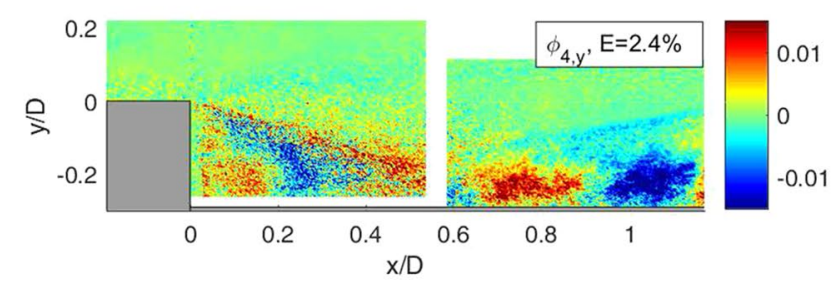

(g)



(i)

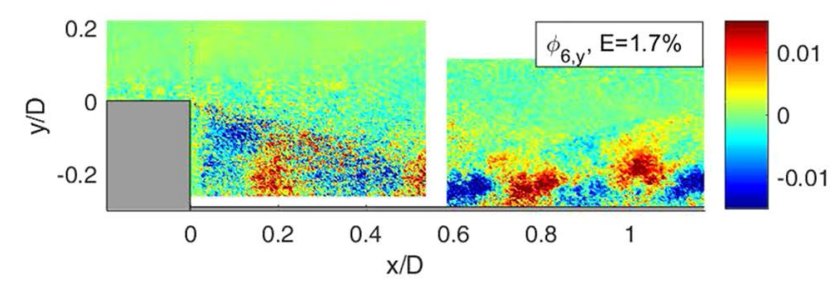

(k)

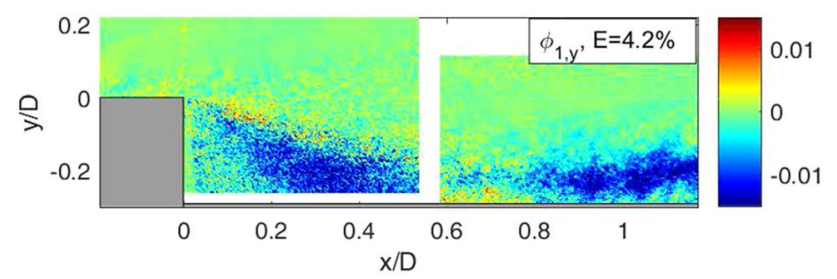

(b)

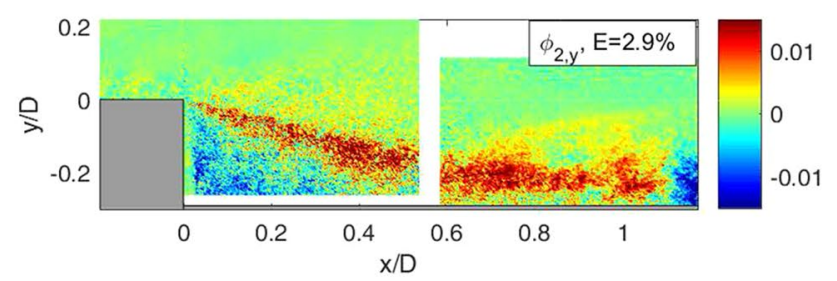

(d)

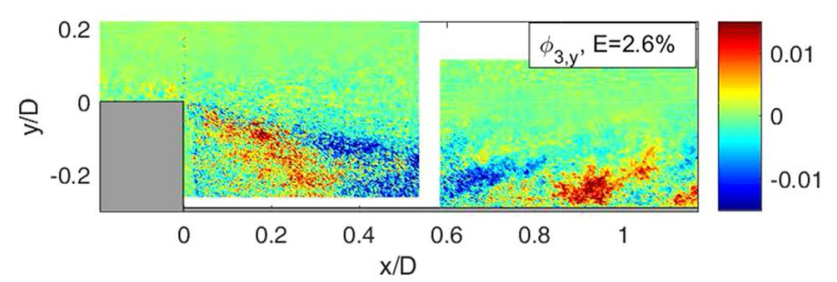

(f)

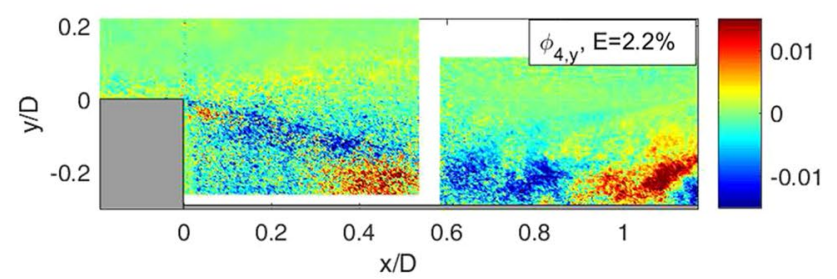

(h)

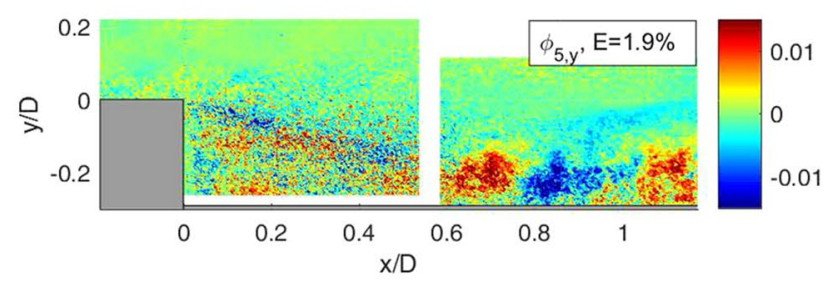

(j)

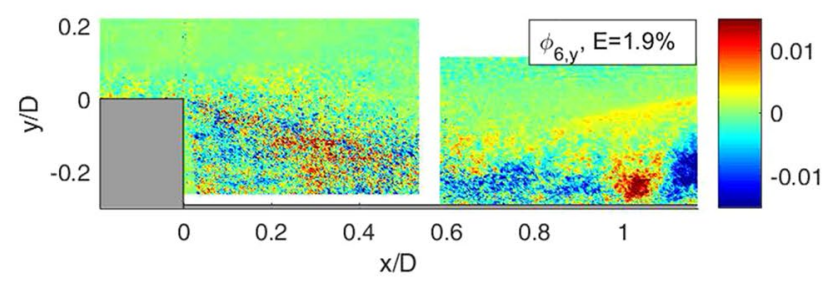

(1)

Fig. 18 POD modes for the radial velocity-fluctuation component $\frac{v}{U_{\infty}}$ without (left column) and with propulsive jet (right column) 
and (b) in the very small coherent turbulent structures in the wake. In the baseline case, on the other hand, a considerable fraction of the energy is contained in the shock motion and low-frequency unsteadiness and the energy content in the turbulent structures is shifted to comparably larger turbulent structures. The POD analysis conducted here thus confirms the assumption of Deprés et al. [1] that the growth of turbulent structures in the shear layer is obstructed by the jet plume.

The observed behavior suggests that if the reattachment process of the flow on the afterbody is completed and the separation region is closed, the instability of the wake is associated with both the shear layer forming downstream of the main body shoulder and the low-frequency unsteadiness of the separation bubble and recompression shock. Since the reattachment process in the propulsivejet case is incomplete due to the displacement effect of the jet plume, the low-frequency unsteadiness plays a smaller role. The instability of the wake then appears to be mostly associated with the shear-layer instability. This second observation is in good agreement with the findings of Statnikov et al. [11]. Studying the same launcher model with hypersonic inflow, they described flapping and swinging motions of the shear layer as the dominant dynamic mechanisms. In their case, the flow did not reattach on the nozzle fairing at all, which is most probably the reason why they did not observe any contribution of the low-frequency unsteadiness. In the wall-pressure spectrum at the main body base (Fig. 15, thin black line), a weak local increase around $S t_{D} \approx 0.55$ can be observed, which agrees reasonably well with the $S t_{D}=0.6$ observed by Statnikov et al. [11] for a swinging motion of the shear layer. With respect to the reattachment zone, the present propulsive-jet case is an intermediate case: the reattachment process starts (as in the baseline case), but is not completed, so that a stable reattached boundary layer is not present at all times (as in the case of Statnikov et al. [11]).

The observation that more energy is contained in the modes associated with the larger turbulent structures in the baseline wake, whereas the much smaller structures contain a larger fraction of the turbulent kinetic energy in the propulsive-jet case, is in good agreement with the observed turbulence levels (see Sect. 3.3). It supports the explanation of Deprés et al. [1] that the physical presence of the propulsive jet obstructs the growth of the structures.

The similarities between the observations in the present supersonic case and the transonic and hypersonic cases studied by Deprés et al. [1] and Statnikov et al. [11], respectively, thus indicate that the governing mechanisms are merely governed by flow geometry, especially the separation topology, and are similar for the different flow regimes relevant for space-launcher trajectories.

\section{Conclusions}

The wake-flow field of a generic axisymmetric spacelauncher model and the influence of an afterexpanding propulsive jet on the flow field were discussed in detail. Measurements were performed in the hypersonic Ludwieg tube Braunschweig at a Mach number of 2.9 and a Reynolds number $\operatorname{Re}_{D}=1.3 \cdot 10^{6}$ based on model diameter $\mathrm{D}$. The nozzle exit velocity of the cold air jet simulating the propulsive jet was at Mach 2.5. I performed velocity measurements in the wake flow by means of particle image velocimetry and wall-pressure measurements on the main body base and nozzle fairing. Based on these data, the evolution of the wake flow was observed along with its spectral content. The mean flow topology and turbulence behavior of the wake were described based on the mean and turbulent velocity fields. A large separated zone forms downstream of the step decrease in diameter at the interface between launcher main body and nozzle fairing. In the baseline case, the flow reattaches downstream onto the nozzle fairing. Under the influence of the jet plume, the flow is displaced away from the wall, the separated region increases in size and the reattachment process is not completed. The propulsive jet appears to have a stabilizing effect on the wake flow: the development of the shear layer and the magnitude of the turbulent intensities and Reynolds shear stresses are damped, and a smaller fraction of the turbulent kinetic energy is associated with the larger coherent structures in the wake. This effect provides evidence to the previously phrased assumption that the physical presence of the jet in the center of the wake obstructs the growth of larger vortical structures in the shear layer. The second main influence of the afterexpanding propulsive jet is effected via its influence on the reattachment process. Without a propulsive jet, full reattachment occurs and a closed separation bubble forms. In this case, the lowfrequency unsteadiness of the separation bubble and recompression shock contributes strongly to the unsteadiness mechanisms of the wake, as discussed based on a POD of the velocity fields. This mechanism has only a minor influence in the propulsive-jet case, where the reattachment process is not completed. In this case, the shear-layer instabilities dominate the dynamic behavior of the wake. The PSD of the wall-pressure signal supports these findings. The prominent local intensity peak around $\mathrm{St}_{D}=0.21$, corresponding to the interaction between vortices shed from the separation bubble and the wall, is much weaker for the incomplete reattachment in the propulsive-jet case. There, on the other hand, a weak local intensity maximum around $\mathrm{St}_{D} \approx 0.55$ can be observed, which is typical for a swinging motion of the shear layer. Similar behavior has been observed in transonic and hypersonic wake flows. The governing mechanisms in launcher-wake flows thus appear to be merely governed by 
the flow geometry, especially the properties of the separation region, and are similar over a large range of flow regimes.

Acknowledgements The German Research Foundation DFG is gratefully acknowledged for funding this research in the framework of the Collaborative Research Centre SFB-TR40 "Technological foundations for the design of thermally and mechanically highly loaded components of future space transportation systems.

The author would like to thank Rolf Radespiel for discussing the research and for insightful remarks. Sven Pülm and Sören Stephan are acknowledged for their assistance during the PIV measurement campaign. Open Access funding provided by Projekt DEAL.

Open Access This article is licensed under a Creative Commons Attribution 4.0 International License, which permits use, sharing, adaptation, distribution and reproduction in any medium or format, as long as you give appropriate credit to the original author(s) and the source, provide a link to the Creative Commons licence, and indicate if changes were made. The images or other third party material in this article are included in the article's Creative Commons licence, unless indicated otherwise in a credit line to the material. If material is not included in the article's Creative Commons licence and your intended use is not permitted by statutory regulation or exceeds the permitted use, you will need to obtain permission directly from the copyright holder. To view a copy of this licence, visit http://creativecommons.org/licenses/by/4.0/.

\section{References}

1. Deprés, D., Reijasse, P., Dussauge, J.-P.: Analysis of unsteadiness in afterbody transonic flows. AIAA J. 42(12), 2541-2550 (2004). https://doi.org/10.2514/1.7000

2. Deck, S., Thorigny, P.: Unsteadiness of an axisymmetric separating-reattaching flow: numerical investigation. Phys. Fluids 19(6), 065103-1-065103-20 (2007). https://doi.org/10.1063/1.2734996

3. Weiss, P.-É., Deck, S., Robinet, J.-C., Sagaut, P.: On the dynamics of axisymmetric turbulent separating/reattaching flows. Phys. Fluids 21(7), 075103-1-075103-8 (2009). https://doi.org/10.1063/1.3177352

4. Bannink, W.J., Houtman, E.M., Bakker, P.G.: Base flow/underexpanded exhaust plume interaction in a supersonic external flow, A98-1598, 8th AIAA International Space Planes and Hypersonic Systems and Technologies Conference, 1998. xx xx, xx (1998). https://doi.org/10.2514/6.1998-1598

5. Bourdon, C.J., Dutton, J.C.: Planar visualizations of largescale turbulent structures in axisymmetric supersonic separated flows. Phys. Fluids 11(1), 201-213 (1999). https://doi. org/10.1063/1.869913

6. Janssen, J.R., Dutton, J.C.: Time-series analysis of supersonic base-pressure fluctuations. AIAA J. 42(3), 605-613 (2004). https ://doi.org/10.2514/1.4071

7. Stephan, S., Wu, J., Radespiel, R.: Propulsive jet influence on generic launcher base flow. CEAS Space J. 7(4), 1-21 (2015). https://doi.org/10.1007/s12567-015-0098-9

8. Stephan, S., Radespiel, R.: Propulsive jet simulation with air and helium in launcher wake flows. CEAS Space J. 9(2), 195-209 (2017). https://doi.org/10.1007/s12567-016-0142-4

9. Saile, D., Gülhan, A., Henckels, A., Glatzer, C., Statnikov, V., Meinke, M.: Investigations on the turbulent wake of a generic space launcher geometry in the hypersonic flow regime. EUCASS Progr. Flight Phys. 5, 209-234 (2013). https://doi.org/10.1051/ eucass/201305209

10. Saile, D., Gülhan, A.: Plume-induced effects on the near-wake region of a generic space launcher geometry, 32nd AIAA applied aerodynamics conference (AIAA Aviation). (2014). https://doi. org/10.2514/6.2014-3137

11. Statnikov, V., Sayadi, T., Meinke, M., Schmid, P., Schröder, W.: Analysis of pressure perturbation sources on a generic space launcher after-body in supersonic flow using zonal turbulence modeling and dynamic mode decomposition. Phys. Fluids 27(1), 016103-1-016103-21 (2015). https://doi.org/10.1063/1.4906219

12. van Gent, P., Payanda, Q., Brust, S., van Oudheusden, B.W., Schrijer, F.J.: Experimental study of the effects of exhaust plume and nozzle length on transonic and supersonic axisymmetric base flows. 7th European Conference for Aeronautics ans Space Sciences (EUCASS), 2017, Milan, Italy

13. Wu, J., Zamre, P., Radespiel, R.: Disturbance characterization and flow quality improvement in a tandem nozzle Mach 3 wind tunnel. Exp. Fluids 56(20), 1-18 (2015). https://doi.org/10.1007/s0034 8-014-1887-1

14. Casper, M., Stephan, S., Scholz, P., Radespiel, R.: Qualification of oil-based tracer particles for heated Ludwieg tubes. Exp. Fluids 55, 1-15 (2014). https://doi.org/10.1007/s00348-014-1753-1

15. Angele, K.P., Muhammad-Klingmann, B.: A simple model for the effect of peak-locking on the accuracy of boundary layer turbulence statistics in digital PIV. Exp. Fluids 38(3), 341-347 (2005). https://doi.org/10.1007/s00348-004-0908-x

16. Piponniau, S., Dussauge, J.-P., Debiève, J.-F., Dupont, P.: A simple model for low-frequency unsteadiness in shock-induced separation. J. Fluid Mech. 629, 87-108 (2009). https://doi. org/10.1017/S0022112009006417

17. Smits, A.J., Hayakawa, K., Muck, K.-C.: Constant temperature hot-wire anemometer practice in supersonic flows. Part 1: the normal wire. Exp. Fluids 1(2), 83-92 (1983). https://doi.org/10.1007/ BF00266260

18. Schreyer, A.-M., Stephan, S., Radespiel, R.: Characterization of the supersonic wake of a generic space launcher. CEAS Space J. 9(1), 97-110 (2017). https://doi.org/10.1007/s12567-016-0134-4

19. Statnikov, V., Stephan, S., Pausch, K., Meinke, M., Radespiel, R., Schröder, W., Experimental and numerical investigations of the turbulent wake flow of a generic space launcher at $M=3$ and $M=6$, 6th European Conference for Aeronautics and Space Sciences (EUCASS): Krakow. Poland (2015). https://doi.org/10.1007/ s12567-016-0112-x

20. Lumley, J.L.: The structure of inhomogeneous turbulent flows. In: Yaglom und A.M., Tatarsky (Hg.) V.I.: Atmospheric turbulence and radio wave propagation - Proceedings of the international colloquium. Moscow, June 15-22 1965. Moscow, Russia, Nauka, 1967, pp. 166-176

21. Berkooz, G., Holmes, P., Lumley, J.L.: The proper orthogonal decomposition in the analysis of turbulent flows. Ann. Rev. Fluid Mech. 25, 539-575 (1993). https://doi.org/10.1146/annur ev.fl.25.010193.002543

22. Sirovich, L.: Turbulence and the dynamics of coherent structures. Part I: Coherent structures. Q. Appl. Math. XLV(3), 561-571 (1987). https://doi.org/10.1090/qam/910462

23. Schreyer, A.-M.: Influence of a propulsive jet on the wake-flow structure of an axisymmetric space-launcher model: 4D-2, 10th international symposium on turbulence and shear flow phenomena (TSFP10), (2017), Chicago, USA

24. Clemens, N.T., Narayanaswamy, V.: Low-frequency unsteadiness of shock wave/turbulent boundary layer interactions. Ann. Rev. Fluid Mech. 46, 469-492 (2014). https://doi.org/10.1146/annur ev-fluid-010313-141346

Publisher's Note Springer Nature remains neutral with regard to jurisdictional claims in published maps and institutional affiliations. 